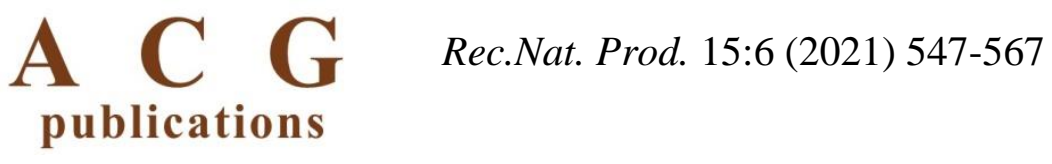

records of natural products

\title{
Antimicrobial, Cytotoxic, Antiviral Effects, and Spectroscopic Characterization of Metabolites Produced by Fusarium oxysporum YP9B
}

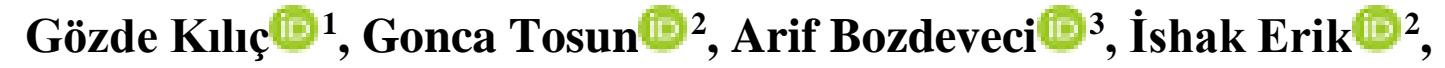 Elif Öztürk ${ }^{4}$, Rengin Reis ${ }^{5,6}$, Hande Sipahi $\odot^{5}$, Merve Cora ${ }^{7}$, Şengül Alpay Karaoğlu $\odot{ }^{3, *}$ and Nurettin Yaylı $\odot$,**}

\author{
${ }^{I}$ Institute of Health Sciences, Karadeniz Technical University, Trabzon, Türkiye \\ ${ }^{2}$ Department of Pharmacognosy, Faculty of Pharmacy, Karadeniz Technical University, Trabzon, Türkiye \\ ${ }^{3}$ Department of Biology, Faculty of Science, Recep Tayyip Erdoğan University, Rize Türkiye \\ ${ }^{4}$ Departments of Nutrition and Dietetics, Faculty of Health Sciences, Karadeniz Technical University, \\ Trabzon, Türkiye \\ ${ }^{5}$ Deparment of Toxicology, Faculty of Pharmacy, Yeditepe University, Istanbul, Türkiye \\ ${ }^{6}$ Deparment of Toxicology, Faculty of Pharmacy, Acibadem Mehmet Ali Aydinlar University, İstanbul, \\ Türkiye \\ ${ }^{7}$ Department of Medical Microbiology, Karadeniz Technical University School of Medicine, Trabzon, \\ Türkiye
}

(Received June 08, 2020; Revised October 21, 2020; Accepted October 25, 2020)

\begin{abstract}
The goal of the work is to determine the bioactive pharmaceutical metabolites produced by the Fusarium oxysporum YP9B isolate. Ten new natural compounds were isolated from the ethyl acetate extract of the $F$. oxysporum YP9B strain. Their structures were elucidated by spectroscopic methods using 1D and 2D NMR, UV, FT-IR, and mass spectra (LC-QTOF MS and GC-FID/MS). Identified compounds were named as; (1-benzyl-2-methoxy-2oxoethyl)-2-hydroxy-3-methylbutanoate (1), 2-oxo-8-azatricyclo[9.3.1.1 $\left.{ }^{3,7}\right]$-hexadeca-1(15),3(16),4,6,11,13-hexaen10-one (2), 2,3-dihydroxypropanoic, hexadecanoic anhydride (3a), 2,3-dihydroxypropanoic (9Z)-octadecenoic anhydride (3b), 2,3-dihydroxy-propanoic (9Z,12Z)-octadecadienoic anhydride (3c), 2,3-dihydroxypropanoic (11Z)octadecenoic anhydride (4a), 2,3-dihydroxypropanoic, (9E,12E)-octadecadienoic anhydride (4b), 3-hydroxy-1,2,6,10tetramethylundecyl hexzadecanoate (5a), 3-hydroxy-1,2,6,10-tetramethylundecyl $(9 E)$-octadecaenoate $(\mathbf{5 b})$, and 3hydroxy-1,2,6,10-tetramethylundecyl octadecanoate (5c). Antimicrobial activities of the isolates obtained from the YP9B strain were determined. Cytotoxic and antiviral activities were tested for the isolates against VERO, MCF-7, PC-3, and A549. Compounds 5a-c, 1, and 3a-c showed bacteriostatic activity at low concentrations, and 4a-b and $\mathbf{2}$ were found to be bactericides. MIC and MBC values against Mycobacterium smegmatis for the compounds 5a-c and 1 were determined to be $<0.5 \mu \mathrm{g} / \mathrm{mL}$ and $0.46 \mu \mathrm{g} / \mathrm{mL}$, respectively. The experimental result showed that compounds 2, 5a-c and 1 have strong cytotoxic $\left(7.51 \pm 1.38\right.$ and $\left.19.13 \pm 0.68(\mu \mathrm{M}) \mathrm{IC}_{50}\right)$ activity. The antiviral activity against HSV type- 1 was determined to be $1.25 \mu \mathrm{M}$ for compounds $\mathbf{4 a - c}$ and $0.312 \mu \mathrm{M}$ for compound $\mathbf{1}$.
\end{abstract}

Keywords: Fusarium oxysporum YP9B; seconder metabolite; antimicrobial; cytotoxic; antiviral. (C) 2021 ACG Publications. All rights reserved.

*Corresponding authors: E-mail address: yayli@ktu.edu.tr (N.Yayl1); Phone: 05333153187; sengulalpay@yahoo.com (Ş.A.Karaoğlu) 
New metabolites produced by Fusarium oxysporum YP9B

\section{Introduction}

The use of fungi for commercial production is ancient, but it has increased rapidly over the last 50 years [1-3]. The pathogenic/nonpathogenic species of fungi are known to release volatile or nonvolatile metabolites in their presence. Numbers of Fusarium species with and without plant pathogens have been identified [2]. The majority of them have been found to secrete mycotoxins, and different chemical structures [4] of these toxins continue to be elucidated. Mushrooms have broad biodiversity. Various interdisciplinary studies were ongoing on the secondary metabolites secreted by nonpathogenic Fusarium species such as $F$. oxysporum, Fusarium graminearum, and Fusarium moniliforme [4-19]. Many studies have focused on the toxicity and diversity of Fusarium species on cereals [20-22]. However, there is little information about secondary metabolites produced by Fusarium that infects fruit-bearing plants rather than cereals [23-24]. In the literature, Alternaria sp., Sinopodophyllum, and $F$. oxysporum were endophyte fungi isolated from Sabirna recurva [14, 25]. In a study, camptothecin type two analogs (9-methoxycamptothecin and 10-hydrocycamptothecin) were reported as anti-cancer microbial products [26]. These microbial products were determined to be produced by the endophyte fungus Fusarium solani, isolated from Camptotheca acuminate. Many secondary metabolites produced by many Fusarium species cause different physiological and pharmacological responses in plants and animals. It is well known that Fusarium species produce trichothecene mycotoxins [4], but these species are also known to have as other compounds; pigments, antibiotics, phytotoxins, etc. [5-19, 27].

In our study, $F$. oxysporum YP9B is isolated from garden soil where vegetables are grown in Pazar-Rize (Eastern Black Sea region of Turkiye). YP9B isolate was determined to produce metabolites containing antimicrobial, cytotoxic and antiviral activities. Details of the isolation, structure elucidation of ten new compounds, and biological activities are presented herein.

\section{Materials and Methods}

\subsection{General}

Optical rotations were measured on Automatic AA-5 Series polarimetry. UV spectra were obtained with a Spectrostar nano BMG labtech spectrometer. Infrared spectra were obtained with a PerkinElmer 1600 FT-IR $\left(4000-400 \mathrm{~cm}^{-1}\right)$ spectrometer. The mass spectral analyses were carried out on an Agilent 6230A LC-Q-TOF-Q-MS. Shimadzu QP2010 ultra GC-FID/MS was used to identify the FAMEs. Melting points were determined using the Thermo-var apparatus fitted with a microscope and are uncorrected. ${ }^{1} \mathrm{H}$ and ${ }^{13} \mathrm{C}$ NMR, along with 2D NMR spectra, were obtained on a Bruker 400 MHz NMR spectrometer (400 MHz for ${ }^{1} \mathrm{H}, 100 \mathrm{MHz}$ for ${ }^{13} \mathrm{C}$ ), using TMS as an internal standard. $\mathrm{CDCl}_{3}$ and acetone- $\mathrm{d}_{6}$ were used as NMR solvent. ACD NMR program was used for the elucidation of isolated compounds. Some of the carbon peaks may exchange in the NMR spectra of compounds $\mathbf{3}$, $\mathbf{4}$, and $\mathbf{5}$ series, which were overlapped due to each series' mixture. Chemical shifts were expressed in $\delta(\mathrm{ppm})$, and coupling constants $(J)$ were reported in hertz $(\mathrm{Hz})$. TLC was carried out on Silica gel $60 \mathrm{~F}_{254}$, and the spots were visualized by spraying with $20 \% \mathrm{H}_{2} \mathrm{SO}_{4}$ and heating. Silica gel was used for column chromatography. Routine laboratory equipment's used were: Refrigerated Centrifuge (Sigma), pH meter (Hanna), Shaker (GFL), Thermoblok (Nosheng), Incubator (Memmert), Shaking oven (Nüve), Gel Imaging System (Uvp), Centrifugal (Sartorius), Bidistilled water (Gfl), Device Security cabinet (Nüve), Power supply (Owl), Autoclave (Nüve),Analytical balance (Denver), and Horizontal Shaker (Nüve).

\subsection{Substrate and Chemicals}

The substrate agar, PDA, yeast extract, Müller Hilton agar, Müller Hilton broth, peptone, corn meal, malt extract agar, $\mathrm{Mg}_{2} \mathrm{SO}_{4}, \mathrm{Na}_{2} \mathrm{SO}_{4}, \mathrm{NaCl}$, sodium acetate, $\mathrm{NaOH}, \mathrm{KH}_{2} \mathrm{PO}_{4} .3 \mathrm{H}_{2} \mathrm{O}, \mathrm{K}_{2} \mathrm{HPO}_{4}$, glucose, dichloran, rose bengal, chloramphenicol (DRBC), agar, $\mathrm{HCl}$, glycerol, ethanol, ethyl acetate, 
chloroform, acetone, $n$-hexane, methanol, silica gel, agarose, yeast nitrogen base, and yeast carbon base were purchased from by Sigma-Aldrich, Fluka or Merck unless otherwise stated. They were in analytical grade unless otherwise stated.

\subsection{Molecular Diagnosis, Revitalization and Extraction from Fusarium sp. YP9B Strain}

The YP9B strain used in the study was isolated from the tomato plant root in Pazar-Rize, Turkey, in August 2014 [28]. The isolated Fusarium sp. is coded as YP9B regarding the region it was isolated (Highway edge in town of Pazar-Rize) [29]. YP9B isolation and characterization were done in Recep Tayyip Erdoğan University Microbiology and Molecular Biology Research Laboratory [30]. The most crucial criterion in the identification of Fusarium species is morphological features, and traditional diagnosis has been made according to the literature information $[28,30]$. To confirm the conventional diagnosis, $F$. oxysporium YP9B has been identified by molecular diagnosis using ITS sequence analysis (18S rRNA ITS1-5.8S-ITS2 intragenic gene regions) and NCBI database (version 5) [31]. Fusarium sp. is defined as YP9B strain, which was before characterized according to the molecular method [31]. F. oxysporum YP9B was determined to be 99\% similarity (GB code MT539140) compared to the sequences existing in GenBank (Table 1).

Table 1. Molecular diagnosis of Fusarium isolates according to 18S rRNA analysis [29]

\begin{tabular}{llccc}
\hline Isolate & \multicolumn{1}{c}{ ITS 1-5.8S-ITS 2 } & Overlap (\%) & Similarity (\%) & Genbank no \\
\hline \multirow{4}{*}{ YP9B } & F. oxysporum strain GFR32 & $93 \%$ & $99.45 \%$ & MT447537.1 \\
& F. oxysporum strain EECC-643 & $93 \%$ & $99.45 \%$ & KP942940.1 \\
& Fusarium sp. strain P1704 & $93 \%$ & $99.45 \%$ & KT268977.1 \\
& F. oxysporum isolate FUS-33 & $93 \%$ & $99.45 \%$ & MH879861.1 \\
& F. oxysporum isolate 107 & $94 \%$ & $99.27 \%$ & KU847855.1 \\
\hline
\end{tabular}

F. oxysporium YP9B isolate was cultured on a plate of potato dextrose agar at $28{ }^{\circ} \mathrm{C}$ for ten days. Potato dextrose agar (PDA) included of Potato Infusion 4.0 G/L (Nitrogen Content 3.0-3.4 \% and Amino-N 2.0-2.2\%), D-(+)-Glucose 20.0 G/L and Agar-Agar 15.0 G/L). The media's final pH was adjusted to $5.6 \pm 0.2\left(25^{\circ} \mathrm{C}\right)$ before sterilization [32]. The YP9B strain was taken from the freezer (at $80^{\circ} \mathrm{C}$ ) was cultivated at $28^{\circ} \mathrm{C}$ for a week by sowing $100 \mu \mathrm{L}$ to the potato dextrose agar (PDA) medium. Fresh cultivation (inoculation) was performed in spot cultivation (3-4 dots) in the plates obtained from PDA agar prepared as a total volume of $10 \mathrm{~L}$. Cultures were incubated for ten days at $28{ }^{\circ} \mathrm{C}$ in an incubator. Later agar plates were cut into small pieces with a sterile scalpel and taken into an Erlenmeyer flask $(2 \mathrm{~L})$ with a maximum of $500 \mathrm{~g}$ isolate with ethyl acetate (1:1 ratio). The media of F. oxisporium YP9B was then incubated at $25^{\circ} \mathrm{C}$ on a rotary shaker at $200 \mathrm{rpm}$ for 48 hours. After the extraction, it was filtered with sterile filter paper and kept at $-20^{\circ} \mathrm{C}$ until it was used.

\subsection{Secondary Metabolite Isolation}

The ethyl acetate extract $(6 \mathrm{~L})$ was completely evaporated under vacuum to afford the crude extract $(5 \mathrm{~g})$. The crude extract $(4.5 \mathrm{~g})$ was subjected to Silica gel $(230-400 \mathrm{mesh})$ column chromatography using $n$-hexane $(100 \mathrm{~mL}), n$-hexane-chloroform $(1: 1100 \mathrm{~mL})$, chloroform $(100 \mathrm{~mL})$, chloroform-ethyl acetate $(1: 1,100 \mathrm{~mL})$, ethyl acetate $(100 \mathrm{~mL})$, ethyl acetate-methanol $(9: 1 ; 8: 2,7: 3$; $6: 4 ; 5: 5 ; 4: 6 ; 3: 7 ; 2: 8$; and $1: 9 ; 100 \mathrm{~mL}$, each), and methanol $(100 \mathrm{~mL})$ gradient elution to afford 30 fractions ( $\sim 50 \mathrm{~mL}$ each). Fractions were control by TLC and were visualized by UV lamp (254 $\mathrm{nm})$ and spraying with $20 \% \mathrm{H}_{2} \mathrm{SO}_{4}$ and heating. In this context, isolated 10 new compounds were obtained from the fractions as follows; compound $\mathbf{1}(80.6 \mathrm{mg})$ from the fraction 11, compound $\mathbf{2}(17.8 \mathrm{mg})$ from the fraction 5, compounds 3a-c (194.2 $\mathrm{mg})$ from the fraction 2, compounds $\mathbf{4 a - b}(61.7 \mathrm{mg})$ from the fraction 3, and compounds 5a-c $(151.0 \mathrm{mg})$ from the fraction 9 within the scope of the work. 
New metabolites produced by Fusarium oxysporum YP9B

\subsection{Preparation of Fatty Acid Methyl Esters (FAMEs)}

Compounds 3a-c, 4a-b, and 5a-c ( $15 \mathrm{mg}$ each) were heated with $5 \%$ sodium hydroxide solution in methanol $(2 \mathrm{~mL})$ at $70^{\circ} \mathrm{C}$ for $3 \mathrm{~h}$. The solutions were cooled, and the aqueous mixture was neutralized with $2 \mathrm{~N} \mathrm{HCl}$ and extracted with diethyl ether $(2 \mathrm{~mL} x 3)$. The organic layer was separated and washed with water $(2 \mathrm{~mL})$, dried over anhydrous $\mathrm{Na}_{2} \mathrm{SO}_{4}$, filtered, and the solvent evaporated. Approximately 5-6 $\mathrm{mg}$ of each sample was dissolved in methanol $(2 \mathrm{~mL})$ in a test tube, and the solutions were cooled in an ice bath; an excess of $\mathrm{BBr}_{3}$ was then added, dropwise. The tube was heated in a boiling water bath at $100^{\circ} \mathrm{C}$ for three $\mathrm{h}$ and cooled. Then water $(3 \mathrm{~mL})$ was added, and methanol evaporated. The aqueous layer was extracted with HPLC grade $n$-hexane ( $2 \mathrm{~mL}$ x 2 times), shaking briefly. The $n$-hexane layers were washed with potassium bicarbonate solution $(2 \mathrm{~mL}, 2 \%)$, dried over anhydrous $\mathrm{Na}_{2} \mathrm{SO}_{4}$, and filtered. The organic solvent was removed under reduced pressure in a rotary evaporator to give FAMEs. FAMEs dissolved in HPLC grade $n$-hexane and were directly analyzed with GC-FID/MS [33].

\subsection{Gas Chromatography-Mass Spectrometry (GC-FID/MS)}

FAMEs analysis was carried out on a Shimadzu QP2010 ultra GC-MS, Shimadzu 2010 plus FID, fitted with a PAL AOC-5000 plus autosampler Shimadzu Class-5000 Chromatography Workstation software. The separation was analyzed using a Restek Rxi-5MS capillary column (30 m x $0.25 \mathrm{~mm} \times 0.25 \mu \mathrm{m})($ USA). FAMEs injections to GC-FID/MS was performed in split mode $(1: 30)$ at $230^{\circ} \mathrm{C}$. The FAMEs solution $(1 \mu \mathrm{L})$ in $n$-hexane (HPLC grade) were injected and analyzed with the column held initially at $60^{\circ} \mathrm{C}$ for $2 \mathrm{~min}$ and then increased to $240^{\circ} \mathrm{C}$ with a $3^{\circ} \mathrm{C} / \mathrm{min}$ heating ramp. The oven program was as follows: the initial temperature was $60^{\circ} \mathrm{C}$ for 2 minutes, which was increased to $240^{\circ} \mathrm{C}$ at 3 minutes, the final temperature of $250^{\circ} \mathrm{C}$ was held for 4 minutes. Helium $(99.999 \%)$ was used as carrier gas with a constant flow-rate of $1 \mathrm{~mL} / \mathrm{min}$. Detection was implemented in electronic impact mode (EI); ionization voltage was fixed at $70 \mathrm{eV}$, scan mode $(40-450 \mathrm{~m} / \mathrm{z})$ was used for mass acquisition. Each sample was analyzed and mean reported.

\subsection{Identification of FAMEs}

Retention indices of the FAMEs were determined by the Kovats method using n-alkanes $\left(\mathrm{C}_{6}{ }^{-}\right.$ $\mathrm{C}_{32}$ ) as standards. FAMEs were identified by comparisons with literature RI [33-37] and MS compared to existing analytical standards and matching mass spectral libraries (NIST, Wiley7NL, FFNSC1.2, and W9N11).

\subsection{Antimicrobial Activity Assessment (Agar-well Diffusion Method)}

All test microorganisms were obtained from the Hifzissihha Institute of Refik Saydam (Ankara, Turkey) and were as follows: Escherichia coli ATCC25922, Yersinia pseudotuberculosis ATCC911, Klebsiella pneumonia subsp. pneumonia ATCC13883, Pseudomonas aeruginosa ATCC27853, Staphylococcus aureus ATCC25923, Enterococcus faecalis ATCC29212, Streptococcus mutans RSKK07038, Lactobacillus casei RSK591, Bacillus cereus 702 Roma, and Mycobacterium smegmatis ATCC607 and also Candida albicans ATCC60193, C. tropicalis ATTCC 13803 and Saccharomyces cerevisiae RSKK251 were used to determine their antifungal activities. Mueller Hinton agar-liquid (MHB, MHA) media for Gram-negative and positive bacteria, Brain Heart Infusion agar-liquid (BHIB, BHIA) media for M. smegmatis, MRS agar for lactobacilli and Potato dextrose agar and Malt extract liquid (PDA, MEB) media for fungi were used for the antimicrobial screening.

Isolates were tested against microorganisms for antimicrobial activity by agar well diffusion method [38-39]. From the overnight cultures of the bacteria to be tested, dilutions of approximately McFarland at 0.5 turbidities (about $10^{6-7} \mathrm{cfu} / \mathrm{mL}$ bacteria) (cfu: colony-forming unit) were prepared in MHB, and widespread cultivation was carried out on pre-prepared MHA plates with a sterile swab. 
For yeast-like fungi, McFarland 2.0 dilutions were made using ME broth, and sterile swab smear was applied to the surface of the previously prepared PDA media. On the medium whose cultivation is completed, wells with a $5 \mathrm{~mm}$ diameter were opened at $2 \mathrm{~cm}$ intervals with a sterile glass pipe. $50 \mu \mathrm{L}$ of the filtrate was added to each well from the isolate of the metabolites with their control. Petri dishes containing bacteria were incubated for 24 hours, and Petri dishes containing yeast and M. smegmatis for 48 hours at $35^{\circ} \mathrm{C}$. Lactobacilli and $S$. mutans were incubated for 48 hours in a $5 \% \mathrm{CO}_{2}$ medium. After incubation, their effectiveness was determined by measuring the inhibition zone diameters with a ruler. Ampicillin for bacteria, streptomycin, and fluconazole for fungi was used as standard control drugs. Standard solvents were also used as controls.

\subsubsection{MIC and MBC assay}

The antimicrobial properties of isolated compounds 1-5 were investigated quantitatively in respective broth media by using double microdilution, and the minimal inhibition concentration (MIC) values $(\mu \mathrm{g} / \mathrm{mL})$ were examined [38-39] and used in our previous work [35-37]. The antibacterial and anti-tuberculosis assays were carried out in Mueller-Hinton and Brain Heart Infusion broths at pH 7.2. The microdilution test plates were incubated for $18-48 \mathrm{~h}$ at $35^{\circ} \mathrm{C}$. The anti-lactobacilli and anti-fungal assays were carried out in MRS and Malt extract broths (Merck, Germany) at $\mathrm{pH}$ 6.2, respectively. The microdilution test plates were incubated for $48 \mathrm{~h}$ at $35^{\circ} \mathrm{C}$ in $5 \% \mathrm{CO}_{2}$. The $\mathrm{MIC}$ was defined as the lowest concentration that showed no growth. Ampicillin $(10 \mathrm{mg} / \mathrm{mL})$, streptomycin $10 \mathrm{mg} / \mathrm{mL}$ and fluconazole $(2 \mathrm{mg} / \mathrm{mL})$ were used as standard antibacterial and antifungal drugs, respectively. Dimethyl sulphoxide, with a dilution of 1:10, was used as solvent control. Concentrations (dilutions without microorganism growth) above the MIC value were used to determine the minimal bactericidal concentration values of the extracts. By taking all dilutions above the MIC value $(100 \mu \mathrm{L})$, passages were passaged at suitable agar media and incubated under appropriate conditions. Dilutions without microorganism development were determined as MBC values.

\subsection{Determination of Cytotoxic and Antiviral Activity}

Isolated compounds (1-5) showed potent antibacterial activity and were then examined for cytotoxic and antiviral activity.

\subsubsection{Cytotoxicity Activity}

VERO culture was used for the cytotoxicity activity of the isolated compounds (1-5). VERO cells, which were 80-90\% confluent in Erlenmeyer, were tripinized and counted using trypan blue. $10^{5}$ cells were cultivated in each well of the 96 -well plate with $100 \mu \mathrm{L}$ of growth medium. The cells were incubated for 5-6 hours at $37^{\circ} \mathrm{C}$ in an oven containing $5 \% \mathrm{CO}_{2}$ to hold the cells. At the end of the incubation, 96-well plate dilutions were prepared, with specific substances and three wells from each concentration. As a negative control, only wells containing cells were used. Prepared plates were incubated at $37^{\circ} \mathrm{C}$ in an oven containing $5 \% \mathrm{CO}_{2}$ for 96 hours. $10 \mu \mathrm{L}$ MTT was added to each well by removing the expired plate. The plate was incubated at $37^{\circ} \mathrm{C}$ in an oven containing $5 \% \mathrm{CO}_{2}$ for 3.5 hours. At the end of incubation, $100 \mu \mathrm{L}$ sterile DMSO was added to the wells by removing the wells' medium. The plates were wrapped in aluminum foil not to see the light and were shaken at room temperature and shaker at low speed for 30 minutes. Plates were read on the spectrophotometer at a wavelength of $570 \mathrm{~nm}$, and the results were evaluated in the Microsoft Excel program regarding the control wells. The viability rate of the cells in the control well was determined to be $100 \%$, and the viability rate of the cells in the wells added to the substance was defined as $\%$.

\subsubsection{Antiviral Activity}

VERO cells, which were $80-90 \%$ confluent in the flask, were counted using trypan blue. $10^{4}$ cells were cultivated in each well of the 96 -well plate with $100 \mu \mathrm{L}$ of growth medium. The cells were 
New metabolites produced by Fusarium oxysporum YP9B

incubated for 5-6 hours at $37^{\circ} \mathrm{C}$ in an oven containing $5 \% \mathrm{CO}_{2}$ to hold the cells. The medium in the wells was evacuated. The number of viruses was adjusted on the medium to be $1 \mathrm{MOI}$ based on the number of infections (MOI), and $100 \mu \mathrm{L}$ of the virus was added to the plate. Plates were removed at $37^{\circ} \mathrm{C}$ for one hour in an oven containing $5 \% \mathrm{CO}_{2}$, with an interval of 10 minutes, and incubated by shaking. The wells' media were emptied, and the concentrations of the substances specified in table 2 were prepared with maintenance medium and placed in the wells with $100 \mu \mathrm{L}$ and three replicates. Acyclovir at a concentration of $25 \mu \mathrm{g} / \mathrm{mL}$ was used as a positive control, and only wells containing virus were used as a negative control. Plates were incubated for three days at $37^{\circ} \mathrm{C}$ in an oven containing $5 \% \mathrm{CO}_{2}$. At the end of the period, $10 \mu \mathrm{L}$ of MTT was added to the wells and incubated for 3.5 hours at $37^{\circ} \mathrm{C}$ in an oven containing $5 \% \mathrm{CO}_{2}$. At the end of the incubation, $100 \mu \mathrm{L}$ DMSO was added to the wells by removing the medium in the wells. The plates were shaken in a dark environment at room temperature for 30 minutes at low speed. Absorbance values of wells were read at $570 \mathrm{~nm}$ in a spectrophotometer. The results were evaluated in the Microsoft Excel program, and the viability rates of the cells were calculated as a percentage. The project was committed to testing against a DNA and an RNA (HSV and polio) virus to determine antiviral activity. Still, only the DNA virus HSV-I (Human herpes simplex virus Type-I) was examined due to a lack of budget.

\subsubsection{Cell Viability Assay}

Cell viability was determined by MTT (3-(4,5-dimethylthiazol-2-yl)-2,5-diphenyltetrazolium bromide) assay. MCF-7 (human breast cancer), PC-3 (human prostate cancer), and A549 (human lung adenocarcinoma) (ATCC, USA) were seeded in a 48-well plate and incubated for $24 \mathrm{~h}$ to form a semiconfluent layer [40-41]. After $24 \mathrm{~h}$, cells were exposed to different concentrations of compounds dissolved in DMSO. After $24 \mathrm{~h}$ incubation, MTT was added to all wells at $0.5 \mathrm{mg} / \mathrm{mL}$ of concentration and incubated an additional $2 \mathrm{~h}$ at $37^{\circ} \mathrm{C}$. After discarding the medium from plates, $100 \mu \mathrm{L}$ of isopropanol was added to the wells. The absorbance of the MTT formazan was determined at $570 \mathrm{~nm}$ by a UV-spectrophotometric plate reader. Viability was defined as the ratio (expressed as a percentage) of the cells' absorbance exposed to compounds to the cells treated with $0.5 \%$ DMSO (v/v). As a reference standard, doxorubicin $\mathrm{HCl}$ was used. All measurements were done in triplicates.

Compound 1: Colorless semi-solid, m.p. $35-38^{\circ} \mathrm{C} ; \mathrm{R}_{\mathrm{f}}$ : 0.90 (ethyl acetate-methanol, 9:1); $[\alpha]_{\mathrm{d}}{ }^{25}-54.86$ (c 0.0056, Ethyl acetate); UV (Ethyl acetate) $\lambda_{\max } \mathrm{nm}(\log \varepsilon): 290$ (2.36); FT-IR (ATR) 3392 (-OH), 3007 (=C-H), 2919 (CH), 1659 (C=O), 1437(C=C), 1407 (C-C), 1315, 1016, 952, 901, $705 \mathrm{~cm}^{-1}$; $\mathrm{C}_{15} \mathrm{H}_{20} \mathrm{O}_{5}$, Positive LC-QTOF-MS: $m / z$ (\%) [M-OCH $\left.3+\mathrm{Na}+2 \mathrm{H}\right]^{+} 274.2987$ (100), calc. 274.2985; for ${ }^{1} \mathrm{H}$ and ${ }^{13} \mathrm{C}$ NMR spectroscopic data see table 2.

Compound 2: Light yellow solid, m.p. $125-127^{\circ} \mathrm{C} ; \mathrm{R}_{\mathrm{f}}: 0.78$ (ethyl acetate); $[\alpha]_{\mathrm{d}}{ }^{25}-4.0$ (c 0.0025 , Ethyl acetate); UV (Ethyl acetate) $\lambda_{\max } \mathrm{nm}(\log \varepsilon): 320$ (2.88); FT-IR (ATR) ): $3355(-\mathrm{NH}), 2926,2855(\mathrm{CH})$, $1673(\mathrm{C}=\mathrm{O}), 1588,1450(\mathrm{C}=\mathrm{C}), 1282,1233(\mathrm{C}-\mathrm{O}), 998,769,692 \mathrm{~cm}^{-1}, \mathrm{C}_{14} \mathrm{H}_{11} \mathrm{NO}_{2}$, Positive LCQTOF-MS: $m / z(\%)\left[\mathrm{M}+\mathrm{CH}_{3} \mathrm{OH}+\mathrm{H}_{2} \mathrm{O}\right]^{+} 274.2815$ (100), calc. 274.2813 ; for ${ }^{1} \mathrm{H}$ and ${ }^{13} \mathrm{C} \mathrm{NMR}$ spectroscopic data see table 3 .

Compounds 3a-c: m.p. (mix.) $165-169^{\circ} \mathrm{C}$; $\mathrm{R}_{\mathrm{f}}$ : 0.86 (chloroform-methanol, 7:2); [ $\left.\alpha\right]_{\mathrm{d}}^{25}+3.89$ (c 0.0077 , $\left.\mathrm{CHCl}_{3}\right) ; \mathrm{UV}\left(\mathrm{CHCl}_{3}\right) \lambda_{\max } \mathrm{nm}(\log \varepsilon): 285$ (1.95), 290 (3.55); FT-IR (ATR) ): 2922, 2853 (CH), 1742 $(\mathrm{C}=\mathrm{O}), 1464,1377(\mathrm{C}=\mathrm{C}), 1161,1097(\mathrm{C}-\mathrm{O}), 972,722,699 \mathrm{~cm}^{-1} ; 3 \mathrm{a}\left(\mathrm{C}_{19} \mathrm{H}_{36} \mathrm{O}_{5}\right)$, Positive LC-QTOFMS: $m / z(\%)\left[\mathrm{M}+\mathrm{CH}_{3} \mathrm{OH}+\mathrm{Na}\right]^{+} 399.2561$ (8), calc. 399.2576; 3b $\left(\mathrm{C}_{21} \mathrm{H}_{38} \mathrm{O}_{5}\right)$, Positive LC-QTOF-MS: $\mathrm{m} / \mathrm{z}(\%)[\mathrm{M}+2 \mathrm{H}+\mathrm{Na}]^{+} 395.2750$ (6), calc. 395.2762, [M-H $\left.\mathrm{H}_{2} \mathrm{O}+\mathrm{H}\right]^{+} 353.2693$ (100), calc. $353.2641 ; 3 \mathrm{c}$ $\left(\mathrm{C}_{21} \mathrm{H}_{36} \mathrm{O}_{5}\right)$, Positive LC-QTOF-MS: $m / z(\%)[\mathrm{M}+2 \mathrm{Na}+\mathrm{H}]^{+} 415.2449$ (10), calc. 415.2435 ; for ${ }^{1} \mathrm{H}$ and ${ }^{13} \mathrm{C}$ NMR spectroscopic data see tables 4 and 5.

Compounds 4a-b: m.p. (mix.) 60-64º ${ }^{\circ}$; $\mathrm{R}_{\mathrm{f}}$ : 0.82 (ethyl acetate); $[\alpha]_{\mathrm{d}}^{25}+8.33$ (c $0.0024, \mathrm{CHCl}_{3}$ ); UV $\left(\mathrm{CHCl}_{3}\right) \lambda_{\max } \mathrm{nm}(\log \varepsilon): 300$ (2.52); FT-IR (ATR) ): 2924, $2854(\mathrm{CH}), 1744(\mathrm{C}=\mathrm{O}), 1464(\mathrm{C}=\mathrm{C}), 1164$ (C-O), 721, $617 \mathrm{~cm}^{-1} ; 4 \mathrm{a}\left(\mathrm{C}_{21} \mathrm{H}_{38} \mathrm{O}_{5}\right)$, Positive LC-QTOF-MS: $\mathrm{m} / z$ (\%) [M+Na] 393.2643 (15), calc. 
393.2616, [M- $\left.\mathrm{H}_{2} \mathrm{O}+\mathrm{H}\right]^{+} 353.2689$ (100), calc. 353.2651; $4 \mathrm{~b}\left(\mathrm{C}_{21} \mathrm{H}_{36} \mathrm{O}_{5}\right)$, Positive LC-QTOF-MS: $\mathrm{m} / \mathrm{z}$ (\%) $\left[\mathrm{M}+\mathrm{H}_{2} \mathrm{O}\right]^{+} 386.2692$ (6), calc. 386.2699, $\left[\mathrm{M}+\mathrm{CH}_{3} \mathrm{OH}\right]^{+} 400.2959$ (8), calc. 400.2962 ; for ${ }^{1} \mathrm{H}$ and ${ }^{13} \mathrm{C}$ NMR spectroscopic data see tables 4 and 5.

Compounds 5a-c: Semi-solid m.p. (mix.) $36-40^{\circ} \mathrm{C}$; $\mathrm{R}_{\mathrm{f}}$ : 0.80 (ethyl acetate-methanol, 9:0.5); $[\alpha]_{\mathrm{d}}{ }^{25}$ +73.33 (c 0.0015, Ethyl acetate); UV (Ethyl acetate) $\lambda_{\max } \mathrm{nm}(\log \varepsilon): 285$ (2.72); FT-IR (ATR): 3394 (-OH), 2964, 2929, $2856(\mathrm{CH}), 1737(\mathrm{C}=\mathrm{O}), 1458,1466(\mathrm{C}=\mathrm{C}), 1188(\mathrm{C}-\mathrm{O}), 1007,618 \mathrm{~cm}^{-1}$; 5a $\left(\mathrm{C}_{31} \mathrm{H}_{62} \mathrm{O}_{3}\right)$, Positive LC-QTOF-MS: $m / z$ (\%) $\left[\mathrm{M}+\mathrm{CH}_{3} \mathrm{OH}+\mathrm{H}\right]^{+} 515.8619$ (5), calc. 515.8610, $\left[\mathrm{M}+2 \mathrm{H}_{2} \mathrm{O}\right]^{+} 518.8499(65)$, calc. $518.8415 ; 5 \mathrm{~b}\left(\mathrm{C}_{33} \mathrm{H}_{64} \mathrm{O}_{3}\right)$, Positive LC-QTOF-MS: $m / z(\%)\left[\mathrm{M}+\mathrm{H}_{2} \mathrm{O}-\right.$ $\mathrm{H}]^{+} 525.8546$ (60), calc. 252.8531; 5c $\left(\mathrm{C}_{33} \mathrm{H}_{66} \mathrm{O}_{3}\right)$, Positive LC-QTOF-MS: $\mathrm{m} / z(\%)[\mathrm{M}]^{+} 510.8655$ (4), calc. 510.8649, $[\mathrm{M}+\mathrm{Na}]^{+} 533.8541$ (4), calc. 533.8546; for ${ }^{1} \mathrm{H}$ and ${ }^{13} \mathrm{C}$ NMR spectroscopic data see tables 6 and 7.

\section{Results and Discussion}

\subsection{Structure Elucidation}

In our study, it was observed that the strains isolated from garden soil in Pazar (Rize) and identified as $F$. oxysporum YP9B by traditional methods [37-38] produce a significant metabolite against antagonistic fungus species used for the biological struggle but do not show any pathogenic features against germination of tomato seeds and vegetable plants. Seconder metabolites produced from Fusarium YP9B strain were extracted with ethyl acetate. The crude extract was purified by column chromatography, a total of 10 new compounds $(\mathbf{1}, \mathbf{2}, \mathbf{3 a}-\mathbf{c}, \mathbf{4 a - b}$, and $\mathbf{5 a - c})$ were isolated, and their structures were identified by spectroscopic methods using NMR (1D: ${ }^{1} \mathrm{H},{ }^{13} \mathrm{C}$, APT and 2D: COSY, TOCSY, HMBC, and HSQC), FT-IR and mass spectra (LC-QTOF-MS, LC-MS and GCFID/MS) and their formulas are given in Figure 1.<smiles>COC(=O)C(Cc1ccccc1)OC(=O)C(O)C(C)C</smiles>

1<smiles>[R]C(=O)OC(=O)C(O)CO</smiles>

3a, $\mathrm{R}=-\mathrm{C}_{15} \mathrm{H}_{31}$

3b, $\mathrm{R}=-\mathrm{C}_{17} \mathrm{H}_{33}\left(\Delta^{9} ; Z\right)$

3c, $\mathrm{R}=-\mathrm{C}_{17} \mathrm{H}_{31}\left(\Delta^{9,12}, Z, Z\right)$

4a, $\mathrm{R}=-\mathrm{C}_{17} \mathrm{H}_{33}\left(\Delta^{11} ; Z\right)$

4b, $\mathrm{R}=-\mathrm{C}_{17} \mathrm{H}_{31}\left(\Delta^{9,12}, E, E\right)$<smiles>O=C1CNc2cccc(c2)Oc2cccc1c2</smiles>

2

Figure 1. Structure of compounds 1, 2, 3a-c, 4a-b, and 5a-c isolated from $F$. oxysporum YP9B strain 
New metabolites produced by Fusarium oxysporum YP9B

Compound 1 was obtained as a colorless semi-solid with a negative optical rotation $[\alpha]_{\mathrm{d}}^{25}-54.86$ (c 0.0056, ethyl acetate). Its LC-MS spectrum showed a pseudo-molecular ion peak at $\mathrm{m} / \mathrm{z} 280.21$ $[\mathrm{M}]^{+}$, and others as $303.88[\mathrm{M}+\mathrm{Na}]^{+}, 319.07[\mathrm{M}+\mathrm{K}]^{+}$. Its molecular formula was determined to be $\mathrm{C}_{15} \mathrm{H}_{20} \mathrm{O}_{5}$ (six degrees of unsaturation) from its LC-MS spectrum. The absorption bands in the FT-IR spectrum of compound 1 indicated the presence of hydroxyl groups $\left(3448 \mathrm{~cm}^{-1}\right)$, carbonyl $\left(1659 \mathrm{~cm}^{-}\right.$ ${ }^{1}$ ), and aromatic double bonds $\left(1437 \mathrm{~cm}^{-1}\right)$. The ${ }^{1} \mathrm{H}$ NMR data (Table 2) for compound 1 exhibited typical signals for an ester with a hydroxyl, methoxy and benzyl type carbon skeleton, including three tertiary methyl groups at $\delta 3.07\left(3 \mathrm{H}, \mathrm{s},-\mathrm{OCH}_{3}\right), 0.97\left(3 \mathrm{H}, \mathrm{d}, J=6.8, \mathrm{H}_{4}\right)$, and $0.43\left(3 \mathrm{H}, \mathrm{d}, J=6.8, \mathrm{H}_{5}\right)$, one methylene at $\delta 3.26-3.31$ and 3.03-3.10 $\left(2 \mathrm{H}, \mathrm{dd}, J=4.9\right.$ and $\left.14.3 \mathrm{~Hz},-\mathrm{CH}_{2} \mathrm{Ph}\right)$, and three $\mathrm{CH}$ protons at $\delta .44-5.48\left(1 \mathrm{H}, \mathrm{dd}, J=4.76\right.$ and $\left.14.3 \mathrm{~Hz}, \mathrm{H}_{1^{\prime}}\right), 5.01\left(1 \mathrm{H}, \mathrm{d}, J=8.7 \mathrm{~Hz}, \mathrm{H}_{2}\right)$, and $1.97\left(1 \mathrm{H}\right.$, octet, $\left.\mathrm{H}_{3}\right)$. Besides, five aromatic protons at $\delta 7.26\left(2 \mathrm{H}, \mathrm{d}, J=7.7 \mathrm{~Hz}, \mathrm{H}_{2^{\prime \prime}, 6^{\prime \prime}}\right), 7.30\left(2 \mathrm{H}, \mathrm{d}, J=7.7 \mathrm{~Hz}, \mathrm{H}_{3^{\prime \prime}, 5^{\prime \prime}}\right)$, and $7.17\left(1 \mathrm{H}, \mathrm{t}, J=7.7 \mathrm{~Hz}, \mathrm{H}_{4^{\prime \prime}}\right)$ were also observed in the ${ }^{1} \mathrm{H}$ NMR spectrum of compound 1 . The $2 \mathrm{D}{ }^{1} \mathrm{H}-$ ${ }^{1} \mathrm{H}$ COSY and TOCSY NMR data of compound 1 showed the $\mathrm{H}_{2}$ to $\mathrm{H}_{5}, \mathrm{H}_{1^{\prime}}$ to $-\mathrm{CH}_{2} \mathrm{Ph}$ and aromatic phenyl $\mathrm{H}_{2^{\prime \prime}, 6^{\prime \prime}}, \mathrm{H}_{3^{\prime \prime}, 5^{\prime \prime}}$, and $\mathrm{H}_{4 "}$ correlation (Table 2).

The ${ }^{13} \mathrm{C}$ NMR spectrum of compound $\mathbf{1}$, in combination with the APT, ${ }^{13} \mathrm{C}-{ }^{1} \mathrm{H}$ HSQC, and HMBC spectra, showed 13 carbon signals that were classified into three methyl at $\delta 19.65\left(\mathrm{C}_{4}\right), 17.71$ $\left(\mathrm{C}_{5}\right)$, and $57.07\left(-\mathrm{OCH}_{3}\right)$, one methylene at $\delta 34.54\left(-\mathrm{CH}_{2} \mathrm{Ph}\right)$, six methines at $\delta 74.78\left(\mathrm{C}_{2}\right), 31.78\left(\mathrm{C}_{3}\right)$, $74.54\left(\mathrm{C}_{1^{\prime}}\right), 128.35\left(\mathrm{C}_{2^{\prime \prime}, 6^{\prime \prime}}\right), 129.07\left(\mathrm{C}_{3^{\prime \prime}, 5^{\prime \prime}}\right)$, and $126.54\left(\mathrm{C}_{4^{\prime \prime}}\right)$, and three quaternary carbons at $\delta 169.79$ $\left(\mathrm{C}_{1}\right), 169.02\left(\mathrm{C}_{2^{\prime}}\right)$, and $137.27\left(\mathrm{C}_{1^{\prime \prime}}\right)$ (Table 2$)$.

Table 2. NMR data of compound $1\left(400 \mathrm{MHz}\right.$, Acetone- $\left.\mathrm{d}_{6}\right)$

\begin{tabular}{|c|c|c|c|c|}
\hline \multicolumn{5}{|c|}{$\mathbf{1}\left(\delta_{\mathrm{H}:}: \mathrm{ppm}, J=\mathrm{Hz}\right)^{\mathrm{a}},\left(\delta_{\mathrm{C}}: \mathrm{ppm}\right)^{\mathrm{a}}$} \\
\hline No & ${ }^{1} \mathbf{H}$ & ${ }^{13} \mathrm{C}$ & APT & COSY, TOCSY \\
\hline 1 & - & 169.79 & $\mathrm{COO}$ & - \\
\hline 2 & $5.01, \mathrm{~d}, J=8.7$ & 74.78 & $\mathrm{CH}$ & $\mathrm{H}_{2}-\mathrm{H}_{3}-\mathrm{H}_{4}-\mathrm{H}_{5}$ \\
\hline 3 & $1.97, \mathrm{~m}$ & 31.78 & $\mathrm{CH}$ & - \\
\hline 4 & $0.97, \mathrm{~d}, J=6.8$ & 19.65 & $\mathrm{CH}_{3}$ & - \\
\hline 5 & $0.43, \mathrm{~d}, J=6.8$ & 17.71 & $\mathrm{CH}_{3}$ & - \\
\hline $1^{\prime}$ & $5.44-5.48, \mathrm{dd}, J=4.8,14.3$ & 74.54 & $\mathrm{CH}$ & $\mathrm{H}_{1^{\prime}-\mathrm{CH}_{2} \mathrm{Ph}}$ \\
\hline $2^{\prime}$ & - & 169.02 & $\mathrm{COO}$ & - \\
\hline $\mathrm{OCH}_{3}$ & $3.07, \mathrm{~s}$ & 57.07 & $\mathrm{OCH}_{3}$ & - \\
\hline $\mathrm{Ph}-\mathrm{CH}_{2}$ & $\begin{array}{l}3.26-3.31, \mathrm{dd}, J=4.9,14.3 \\
3.03-3.10, \mathrm{dd}, J=4.9,11.1\end{array}$ & 34.54 & $\mathrm{CH}_{2} \mathrm{Ph}$ & - \\
\hline 1" & - & 137.27 & $\mathrm{C}$ & \\
\hline 2", 6" & $7.26, \mathrm{~d}, J=7.7$ & 128.35 & $\mathrm{CH}$ & \\
\hline 3", 5" & $7.30, \mathrm{~d}, J=7.7$ & 129.07 & $\mathrm{CH}$ & $\mathrm{ArCH}-\mathrm{CH}_{2} \mathrm{Ph}-\mathrm{H}_{1}$ \\
\hline 4" & $7.17, \mathrm{t}, J=7.7$ & 126.54 & $\mathrm{CH}$ & \\
\hline
\end{tabular}

a2D COSY, HMBC, HSQC, and ACD NMR program is used for interpretation.

Based on the above analysis, the structure of compound 1 was elucidated as (1-benzyl-2methoxy-2-oxoethyl)-2-hydroxy-3-methyl butanoate. Close similarities of the chemical shifts of significant carbon signals for compound 1 with reported values for similar compounds confirmed that compound $\mathbf{1}$ is hydroxyl, methoxy, and benzyl substituted ester compound (Table 2, see supporting information for the spectra, Figure S1-6). In the literature review, no compound was found to be in the structure of compound 1. However, screening a similar compound, there is a natural compound in which another terpenic group was replaced instead of the methoxy group of compound $\mathbf{1}$, which was isolated from Ixeris debilis and I. repens [42]

Compound 2 was also obtained as a light yellow solid with the m.p. $125-127^{\circ} \mathrm{C}$. The LC-MS indicated a molecular formula to be $\mathrm{C}_{14} \mathrm{H}_{11} \mathrm{NO}_{2}\left(226.85(35)[\mathrm{M}+\mathrm{H}]^{+}\right.$, and $\left.250.96(100)[\mathrm{M}+\mathrm{Na}+2 \mathrm{H}]^{+}\right)$ for compound 2 . The absorption bands revealed the secondary amine, carbonyl, and aromatic double bond groups at $3355 \mathrm{~cm}^{-1}, 1673 \mathrm{~cm}^{-1}$, and $1450 \mathrm{~cm}^{-1}$ in the FT-IR spectrum of it, respectively. 
Kılıç et al., Rec. Nat. Prod. 15:6 (2021) 547-567

Table 3. NMR data of compound $2\left(400 \mathrm{MHz}\right.$, Acetone- $\left.\mathrm{d}_{6}\right)$

\begin{tabular}{|c|c|c|c|c|}
\hline \multicolumn{5}{|c|}{$2\left(\delta_{\mathrm{H}}: \mathrm{ppm}, J=\mathrm{Hz}\right)^{\mathrm{a}},\left(\delta_{\mathrm{C}}: \mathrm{ppm}\right)^{\mathrm{a}}$} \\
\hline No & ${ }^{1} \mathbf{H}$ & ${ }^{13} \mathbf{C}$ & APT & COSY \\
\hline 1 & - & 159.22 & $\mathrm{C}$ & \\
\hline 2 & $6.78, \mathrm{bd}, J=2.4$ & 115.30 & $\mathrm{CH}$ & \\
\hline 3 & - & 140.05 & $\mathrm{C}$ & \\
\hline 4 & $6.76, \mathrm{~d}, J=8.8$ & 118.18 & $\mathrm{CH}$ & $\mathrm{H}_{2}-\mathrm{H}_{4}-\mathrm{H}_{5}-\mathrm{H}_{6}$ \\
\hline 5 & $7.12, \mathrm{t}, J=7.8$ & 131.15 & $\mathrm{CH}$ & \\
\hline 6 & $6.96-6.72, \mathrm{dd}, J=8.8,2.3$ & 116.57 & $\mathrm{CH}$ & \\
\hline $1^{\prime}$ & - & 159.42 & $\mathrm{C}$ & \\
\hline $2^{\prime}$ & $7.48, \mathrm{dd}, J=1.8,2.1$ & 121.83 & $\mathrm{CH}$ & \\
\hline $3^{\prime}$ & - & 138.51 & $\mathrm{C}$ & \\
\hline $4^{\prime}$ & $7.56, \mathrm{~d}, J=7.7$ & 122.39 & $\mathrm{CH}$ & $\mathrm{H}_{2}^{\prime}-\mathrm{H}_{4}^{\prime}-\mathrm{H}_{5}^{\prime}-\mathrm{H}_{6}^{\prime}$ \\
\hline $5^{\prime}$ & $7.33, \mathrm{t}, J=7.4$ & 131.48 & $\mathrm{CH}$ & \\
\hline $6^{\prime}$ & $7.05-7.08, \mathrm{dd}, J=0.9,8.0$ & 121.74 & $\mathrm{CH}$ & \\
\hline $1 "$ & - & 196.89 & $\mathrm{C}=\mathrm{O}$ & \\
\hline $2 "$ & $4.22, \mathrm{~s}$ & 45.03 & $\mathrm{CH}_{2}$ & \\
\hline $\mathrm{C}_{6} \mathrm{H}_{5}-\mathrm{NH}$ & $8.60, \mathrm{bs}, 1 \mathrm{H}$ & - & - & \\
\hline
\end{tabular}

Signals for one methylene proton at $\delta 4.22\left(2 \mathrm{H}, \mathrm{s}, \mathrm{H}_{2^{\prime \prime}}\right)$, one $-\mathrm{NH}-$ proton at $\delta 8.60(1 \mathrm{H}$, bs, exchanges with $\left.\mathrm{D}_{2} \mathrm{O}\right)$, and eight aromatic phenyl ring $\mathrm{A}$ and $\mathrm{B}$ protons at $\delta 6.78(1 \mathrm{H}, \mathrm{bd}, J=2.4 \mathrm{~Hz}$, $\left.\mathrm{H}_{2}\right), 6.76\left(1 \mathrm{H}, \mathrm{d}, J=8.8 \mathrm{~Hz}, \mathrm{H}_{4}\right), 7.12\left(1 \mathrm{H}, \mathrm{t}, J=7.8 \mathrm{~Hz}, \mathrm{H}_{5}\right), 6.96-6.72\left(1 \mathrm{H}, \mathrm{dd}, J=2.3\right.$ and $\left.8,8 \mathrm{~Hz}, \mathrm{H}_{6}\right)$ for ring A and at $\delta 7.48\left(1 \mathrm{H}\right.$, dd, $J=1.8$ and $\left.2.1 \mathrm{~Hz}, \mathrm{H}_{2^{\prime}}\right), 7.56\left(1 \mathrm{H}, \mathrm{d}, J=7.7 \mathrm{~Hz}, \mathrm{H}_{4^{\prime}}\right), 7.33(1 \mathrm{H}, \mathrm{t}, J=7.4$ $\left.\mathrm{Hz}, \mathrm{H}_{5^{\prime}}\right), 7.05-7.08\left(1 \mathrm{H}, \mathrm{dd}, J=0.9\right.$ and $\left.8.0 \mathrm{~Hz}, \mathrm{H}_{6^{\prime}}\right)$ for ring B were displayed in the ${ }^{1} \mathrm{H}$ NMR spectrum (Table 3).

The ${ }^{13} \mathrm{C}$ NMR data, together with APT and HSQC experiments, indicated fourteen carbon signals: one methylene at $\delta 45.03\left(\mathrm{C}_{2^{\prime \prime}}\right)$, eight methines at $\delta 115.30\left(\mathrm{C}_{2}\right), 118.18\left(\mathrm{C}_{4}\right), 131.15\left(\mathrm{C}_{5}\right)$, $116.57\left(\mathrm{C}_{6}\right), 121.83\left(\mathrm{C}_{2^{\prime}}\right), 122.39\left(\mathrm{C}_{4^{\prime}}\right), 131.48\left(\mathrm{C}_{5^{\prime}}\right)$, and $121.74\left(\mathrm{C}_{6^{\prime}}\right)$, and five quaternary carbons at $\delta$ 159.22 $\left(\mathrm{C}_{1}\right), 140.05\left(\mathrm{C}_{3}\right), 159.42\left(\mathrm{C}_{1^{\prime}}\right), 138.51\left(\mathrm{C}_{3}\right)$, and $196.89\left(\mathrm{C}=\mathrm{O}, \mathrm{C}_{1^{\prime \prime}}\right)$. All the proton and carbon signals of compound 2 were assigned unambiguously by analyzing its COSY, TOCSY, and HSQC NMR data (Table 3, see supporting information for the spectra, Figure S10-13). Therefore, the structure of compound 2 was in phenoxazine structure and named as 2-oxo-8azatricyclo[9.3.1.1 $1^{3,7}$ hexadeca-1(15),3(16),4,6,11,13-hexaen-10-one. Compound 2 was isolated in a relatively pure form, which is in the tricyclic phenoxazine form. In the literature search, the same compound was not found. However, by scanning similar compounds, some phenoxazine compounds with different link positions have been reported, and their biological activities were also mentioned [43-46].

Compounds 3a-c and 4a-b series are in hydroxyl substituted 2,3-dihydroxypropanoic alky/alkenyl (C16/C18) anhydride, obtained from the different eluate in a mixture of 3a-c and 3a, 4ab compounds. Due to polarity, it was not possible to purify one by one. However, a mixture of compounds 3a-c was hydrolyzed and methylated then GC-FID/MS analyzes were performed as fatty acid methyl ester (FAMEs). As a result of the GC-MS analysis, oleic acid $(49.79 \%, 3 \mathbf{b})$, palmitic acid $(36.86 \%, 3 \mathbf{3})$ and octadecadienoic acid $\left(11.09 \%, 3 \mathbf{c}, 18: 2, \Delta^{9,12},(E, E)\right)$ were characterized. Similarly, mixtures of compounds $\mathbf{3 a}$, and $\mathbf{4 a - b}$ were hydrolyzed and GC-FID/MS analyzes revealed palmitic acid $(\mathbf{3 a}, 63.07 \%)$, octadecanoic acid $\left(\mathbf{4 a}, 34.21 \%, 18: 1, \Delta^{11},(Z)\right)$ and octadecadienoic acid (4b, $\left.2.47 \%, 18: 2, \Delta^{9,12},(E, E)\right)$. Compounds 3a-c and $\mathbf{4 a - b}$ series were mixtures, and their molecular formula was assigned to be $\mathrm{C}_{19} \mathrm{H}_{36} \mathrm{O}_{5}$ for $\mathbf{3 a} ; \mathrm{C}_{21} \mathrm{H}_{38} \mathrm{O}_{5}$ for $\mathbf{3 b} ; \mathrm{C}_{21} \mathrm{H}_{36} \mathrm{O}_{5}$ for $\mathbf{3 c}, \mathrm{C}_{21} \mathrm{H}_{38} \mathrm{O}_{5}$ for $\mathbf{4 a}$; and $\mathrm{C}_{21} \mathrm{H}_{36} \mathrm{O}_{5}$ for $4 \mathbf{b}$ based on LC-QTOF-MS, LC-MS, GC-FID/MS, and NMR data. The ${ }^{1} \mathrm{H}$ and ${ }^{13} \mathrm{C}$ NMR spectra of compounds 3a-c and $\mathbf{4 a - b}$ (Table 4 and 5, see supporting information for the spectra, Figure S17-21, and S25-29) series were very similar. The ${ }^{1} \mathrm{H}$ NMR data for compound 3a-c showed one $\mathrm{CH}$ at $\delta 5.27\left(1 \mathrm{H}, \mathrm{m}, \mathrm{H}_{2^{\prime}}\right)$, one methylene at $\delta 4.27-4.31$ and $4.11-4.16\left(2 \mathrm{H}, \mathrm{m}, \mathrm{H}_{3^{\prime}}\right)$ for 3a-c, one olefin protons at $\delta$ 5.26-5.33 $\left(2 \mathrm{H}, \mathrm{m}, \Delta^{9}\right)$ for $\mathbf{3 b}$ and $\mathbf{3 c}$ (Table 4$)$. The ${ }^{1} \mathrm{H}$ NMR spectrum of compounds $4 \mathbf{4}-$ b gave one $\mathrm{CH}$ at $\delta 5.35\left(2 \mathrm{H}, \mathrm{t}, J=6.28 \mathrm{~Hz}, \mathrm{H}_{2^{\prime}}\right)$ and one methylene at $\delta 4.32-4.36$ and $4.15-4.20(2 \mathrm{H}$, 
New metabolites produced by Fusarium oxysporum YP9B

$\mathrm{dd}, J=3.88$ and $\left.6.28 \mathrm{~Hz}, \mathrm{H}_{3^{\prime}}\right)$ for $\mathbf{4 a}$, and $\mathbf{3 b}$, one olefin protons at $\delta 5.10-5.36\left(2 \mathrm{H}, \mathrm{m}, \Delta^{9}\right)$ for $\mathbf{4 a}$ and two double bond protons at $\delta 5.10-5.36\left(4 \mathrm{H}, \mathrm{m}, \Delta^{9,12}\right)$ for $\mathbf{4 b}$ (Table 4). Other alkyl side chain proton NMR peaks for compounds 3a-c and $\mathbf{4 a - b}$ were in the upfield of spectra at $\delta 0.88-2.32 \mathrm{ppm}$. The ${ }^{13} \mathrm{C}$ NMR spectra of compounds $\mathbf{3 a - c}$ and $\mathbf{4 a - b}$ (Table 5) commonly showed two carbonyls at $\delta 172.12\left(\mathrm{C}_{1}\right)$ and $172.41\left(\mathrm{C}_{1^{\prime}}\right)$, one methyl at $\delta 13.55(\mathrm{C} 16 / \mathrm{C} 18)$, one methylene at $\delta 61.87\left(\mathrm{C}_{3^{\prime}}\right)$, one $\mathrm{CH}$ at $\delta 69.01$ $\left(\mathrm{C}_{2^{\prime}}\right) \mathrm{ppm}$. Other carbon peaks for the alkyl chain are located at $\delta 22.50-33.75 \mathrm{ppm}$. The planar structure of compounds 3a-c and 4a-b were further confirmed by 2D NMR (COSY, HMQC, and HMBC), LCQTOF-MS, and GC-FID/MS spectral analysis and named as 2,3-dihydroxypropanoic hexadecanoic anhydride (3a), 2,3-dihydroxypropanoic (9Z)-octadecenoic anhydride (3b, 2,3-dihydroxypropanoic (9Z,12Z)-octadecadienoic anhydride (3c), 2,3-dihydroxypropanoic (11Z)-octadecenoic anhydride (4a), 2,3-dihydroxypropanoic $(9 E, 12 E)$-octadecadienoic anhydride (4b). According to the literature survey, none of these compounds $\mathbf{3 a}, \mathbf{3 b}, \mathbf{3 c}, \mathbf{4 a}$, and $\mathbf{4 b}$ are known.

Compound 5a-c was the ester type compound containing hydroxyl substituted sesquiterpene units isolated as a mixture. Because of their close polarity, they could not be isolated separately. However, the mixture of compounds 5a-c was hydrolyzed and methylated, then GC-FID/MS analyzes gave palmitic acid $(\mathbf{5 a}, 81.25 \%)$, octadecaenoic acid $\left(\mathbf{5 b}, 12.62 \%, 18: 1, \Delta^{9},(\mathrm{E})\right)$ and stearic acid $(\mathbf{5 c}$, $6.12 \%, 18: 0)$. Compounds 5a-c were a mixture, and their molecular formula was assigned to be $\mathrm{C}_{31} \mathrm{H}_{62} \mathrm{O}_{3}$ for $\mathbf{5 a} ; \mathrm{C}_{33} \mathrm{H}_{64} \mathrm{O}_{3}$ for $\mathbf{5 b}$; and $\mathrm{C}_{33} \mathrm{H}_{66} \mathrm{O}_{3}$ for $\mathbf{5} \mathbf{c}$ LC-QTOF-MS, LC-MS, GC-FID/MS, and NMR data. LC-MS spectrum of 5a-c showed a pseudo-molecular ion peak at $\mathrm{m} / z \quad 482.88[\mathrm{M}]^{+}, 509.06$ $[\mathrm{M}+\mathrm{H}]^{+}$, and $510.75[\mathrm{M}]^{+}$, respectively. The ${ }^{1} \mathrm{H}$ NMR data for compounds $\mathbf{5 a - c}$ showed one $\mathrm{CH}$ at $\delta$ $5.27\left(1 \mathrm{H}, \mathrm{m}, \mathrm{H}_{2^{\prime}}\right)$, one methylene at $\delta 4.27-4.31$ and 4.11-4.16 $\left(2 \mathrm{H}, \mathrm{m}, \mathrm{H}_{3^{\prime}}\right)$ for $\mathbf{5 a - c}$, one olefin protons at $\delta$ 5.26-5.33 $\left(2 \mathrm{H}, \mathrm{m}, \Delta^{9}\right)$ for $5 \mathrm{~b}$ and $5 \mathrm{c}$. The ${ }^{1} \mathrm{H}$ NMR spectrum of compounds 5 gave one $\mathrm{CH}$ at $\delta$ $5.35\left(2 \mathrm{H}, \mathrm{t}, J=6.28 \mathrm{~Hz}, \mathrm{H}_{2^{\prime}}\right)$ and one methylene at $\delta 4.32-4.36$ and $4.15-4.20(2 \mathrm{H}, \mathrm{dd}, J=3.88$ and 6.28 $\left.\mathrm{Hz}, \mathrm{H}_{3^{\prime}}\right)$ for $\mathbf{5 a}$, and $\mathbf{5 b}$, one olefin protons at $\delta 5.10-5.36\left(2 \mathrm{H}, \mathrm{m}, \Delta^{9}\right)$ for $\mathbf{5 a}$ and two double bond protons at $\delta 5.10-5.36\left(4 \mathrm{H}, \mathrm{m}, \Delta^{9,12}\right)$ for $\mathbf{5 b}$. Other alkyl side chain proton NMR peaks for compounds 5a-c were in the upfield of spectra at $\delta 0.88-2.32 \mathrm{ppm}$ (Table 6, see supporting information for the spectra, Figure S33, 36). The ${ }^{13} \mathrm{C}$ NMR spectra of compounds 5a-c commonly showed one carbonyl at $\delta 174.18\left(\mathrm{C}_{1}\right), 170.19\left(\mathrm{C}_{1}\right)$, and $168.94\left(\mathrm{C}_{1}\right)$, one methyl at $\delta 13.61,13.63$, and $14.09(\mathrm{C} 16 / \mathrm{C} 18)$ for alkyl chain, five methyl at $\delta 19.61\left(\mathrm{C}_{10^{\prime}}\right), 18.86\left(\mathrm{C}_{11^{\prime}}\right), 17.94\left(\mathrm{C}_{12^{\prime}}\right), 10.98\left(\mathrm{C}_{13^{\prime}}\right)$, and $17.98\left(\mathrm{C}_{15^{\prime}}\right)$ for the sesquiterpene part, two oxygenated $\mathrm{CH}$ at $\delta 74.65\left(\mathrm{C}_{13^{\prime}}\right), 73.31\left(2 \mathrm{C}, \mathrm{C}_{13^{\prime}}\right)$, and $62.17\left(\mathrm{C}_{2^{\prime}}\right), 62.03\left(\mathrm{C}_{2^{\prime}}\right)$, $61.90\left(\mathrm{C}_{2^{\prime}}\right)$, respectively (Table 7, see supporting information for the spectra, Figure S34-35, 37-38). Other carbon peaks for the alkyl and the sesquiterpene chain of compound 5a-c are located at the upfield of NMR spectra. The planar structure of compounds 5a-c were further confirmed by 2D NMR (COSY, HMQC, and HMBC), LC-QTOF-MS, LC-MS, and GC-FID/MS spectral analysis and named as 3-hydroxy-1,2,6,10-tetramethylundecyl hexzadecanoate, 5a, 3-hydroxy-1,2,6,10tetramethylundecyl (9E)-octadecaenoate, 5b, 3-hydroxy-1,2,6,10-tetramethylundecyl octadecanoate $\mathbf{5 c}$. In the literature review, compounds $\mathbf{5 a}, \mathbf{5 b}$, and $\mathbf{5 c}$ were not found. A literature survey showed that some of the natural compounds contain sesquiterpene part of compounds 5a-c [47-48].

\subsection{Antimicrobial Activities of Compounds 1-5}

Antimicrobial activities of isolated compounds 1-5 were investigated, and minimal inhibition (MIC, $\mu \mathrm{g} / \mathrm{mL}$ ) and minimal bactericidal concentrations (MBC, $\mu \mathrm{g} / \mathrm{mL}$ ) were detected (Table $8 \mathrm{a}, \mathrm{b}$ ). When looking at the antimicrobial activities in general, it was observed that all of the selected fractions had high antimicrobial activity at low concentrations. It was found that the isolated compounds showed high antimicrobial activities, compounds 1, 2, and 5a-c had an anti-microbial reproductive activity, and compounds 4a-c and $\mathbf{2}$ showed a microorganism-killing activity. Compound $\mathbf{1}$ had a strong antimicrobial effect at concentrations of 0.47-1.8 $\mu \mathrm{g} / \mathrm{mL}$ against Gram-positive bacteria (S. aureus, $E$. faecalis, S. mutans, B. cereus, and M. smegmatis) except L. acidophilus, which is a member of normal flora. 
Table 4. ${ }^{1} \mathrm{H}$ NMR data of compounds $\mathbf{3 a}, \mathbf{3 b}, 3 \mathbf{c}, \mathbf{4 a}$, and $\mathbf{4 b}(400 \mathrm{MHz})$

\begin{tabular}{|c|c|c|c|c|c|c|c|c|c|}
\hline \multicolumn{10}{|c|}{ Compounds $\left(\delta_{\mathrm{H}}: \mathrm{ppm}\right)^{\mathrm{a}}$} \\
\hline \multicolumn{6}{|c|}{$\mathrm{CDCl}_{3}$} & \multicolumn{4}{|c|}{ Acetone-d 6} \\
\hline \multicolumn{2}{|c|}{ 3a $(16: 0)$} & \multicolumn{2}{|c|}{ 3b $(18: 1, Z) \Delta^{9}$} & \multicolumn{2}{|c|}{ 3c $(18: 2 ; E, E) \Delta^{9,12}$} & \multicolumn{2}{|c|}{$\mathbf{4 a}(18: 1, Z) \Delta^{11}$} & \multicolumn{2}{|c|}{ 4b $(18: 2 ; E, E) \Delta^{9,12}$} \\
\hline $\mathrm{H}_{2}$ & $1.99-2.02, \mathrm{~m}$ & $\mathrm{H}_{2}$ & $1.99-2.05, \mathrm{~m}$ & $\mathrm{H}_{2}$ & $1.99-2.05, \mathrm{~m}$ & $\mathrm{H}_{2}$ & $2.32, \mathrm{t}, J=7.36$ & $\mathrm{H}_{2}$ & $2.32, \mathrm{t}, J=7.36$ \\
\hline $\mathrm{H}_{16}$ & $0.85-0.88, \mathrm{~m}$ & $\mathrm{H}_{9}-\mathrm{H}_{10}$ & $5.26-5.33, \mathrm{~m}$ & $\mathrm{H}_{9}-\mathrm{H}_{10}$ & $5.26-5.33, \mathrm{~m}$ & $\mathrm{H}_{10}$ & 2.04-2.08, m & $\mathrm{H}_{8}$ & $2.04-2.08, \mathrm{~m}$ \\
\hline- & - & $\mathrm{H}_{11}-\mathrm{H}_{17}$ & $1.25-1.60, \mathrm{~m}$ & $\mathrm{H}_{11}$ & $2.80, \mathrm{~m}$ & $\mathrm{H}_{11}-\mathrm{H}_{12}$ & $5.10-5.36, \mathrm{~m}$ & $\mathrm{H}_{9}-\mathrm{H}_{10}$ & $5.10-5.36, \mathrm{~m}$ \\
\hline- & - & $\mathrm{H}_{18}$ & $0.85-0.88, \mathrm{~m}$ & $\mathrm{H}_{12}-\mathrm{H}_{13}$ & $5.26-5.33, \mathrm{~m}$ & $\mathrm{H}_{13}$ & 2.04-2.08, m & $\mathrm{H}_{11}$ & $2.80, \mathrm{t}, J=6.24$ \\
\hline- & - & - & - & $\mathrm{H}_{18}$ & $0.85-0.88, \mathrm{~m}$ & $\mathrm{H}_{18}$ & $0.88, \mathrm{~m}$ & $\mathrm{H}_{14}$ & 2.04-2.08, m \\
\hline & & & & & & - & - & $\mathrm{H}_{15}-\mathrm{H}_{17}$ & $1.25-1.61, \mathrm{~m}$ \\
\hline & & & & & & - & - & $\mathrm{H}_{18}$ & $0.88, \mathrm{~m}$ \\
\hline $\mathrm{H}_{2}$ & $5.27, \mathrm{~m}$ & $\mathrm{H}_{2}$ & $5.27, \mathrm{~m}$ & $\mathrm{H}_{2}$ & $5.27, \mathrm{~m}$ & $\mathrm{H}_{2}$ & $5.35, \mathrm{t}, J=6.28$ & $\mathrm{H}_{2^{\prime}}$ & $5.35, \mathrm{t}, J=6.28$ \\
\hline $\mathrm{H}_{3^{\prime}}$ & $4.27-4.31, \mathrm{~m}$ & $\mathrm{H}_{3^{\prime}}$ & 4.27-4.31, m & $\mathrm{H}_{3^{\prime}}$ & $4.27-4.31, \mathrm{~m}$ & $\mathrm{H}_{3^{\prime}}$ & $4.32-4.36, \mathrm{dd}, J=3.88$ & $\mathrm{H}_{3^{\prime}}$ & $4.32-4.36, \mathrm{dd}, J=3.88$ \\
\hline & 4.11-4.16, m & & 4.11-4.16, m & & 4.11-4.16, m & & $4.15-4.20, \mathrm{dd}, J=6.28$ & & $4.15-4.20, \mathrm{dd}, J=6.28$ \\
\hline
\end{tabular}


Table 5. ${ }^{13} \mathrm{C}$ NMR data of compounds $\mathbf{3 a}, \mathbf{3 b}, \mathbf{3 c}, \mathbf{4 a}$, and $\mathbf{4 b}(100 \mathrm{MHz})$

\begin{tabular}{|c|c|c|c|c|c|c|c|c|c|c|}
\hline \multicolumn{11}{|c|}{ Compounds $\left(\delta_{\mathrm{C}}: \text { ppm }\right)^{\mathrm{a}}$} \\
\hline \multirow{3}{*}{$\begin{array}{c}\text { No } \\
1\end{array}$} & \multicolumn{6}{|c|}{$\mathrm{CDCl}_{3}$} & \multicolumn{4}{|c|}{$\begin{array}{c}\text { Acetone-d } \\
\end{array}$} \\
\hline & \multicolumn{2}{|c|}{$\mathbf{3 a}(16: 0)$} & \multicolumn{2}{|c|}{ 3b $(18: 1, Z) \Delta^{9}$} & \multicolumn{2}{|c|}{ 3c $(18: 2 ; E, E) \Delta^{9,12}$} & \multicolumn{2}{|c|}{$4 \mathbf{a}(18: 1, Z) \Delta^{11}$} & \multicolumn{2}{|c|}{$4 \mathbf{b}(18: 2 ; E, E) \Delta^{9,12}$} \\
\hline & 172.75 & $\mathrm{C}$ & 173.15 & $\mathrm{C}$ & 173.18 & $\mathrm{C}$ & 172.12 & $\mathrm{C}$ & 172.12 & $\mathrm{C}$ \\
\hline 2 & 33.99 & $\mathrm{CH}_{2}$ & 34.15 & $\mathrm{CH}_{2}$ & 33.99 & $\mathrm{CH}_{2}$ & 33.54 & $\mathrm{CH}_{2}$ & 33.75 & $\mathrm{CH}_{2}$ \\
\hline 3 & 25.61 & $\mathrm{CH}_{2}$ & 24.85 & $\mathrm{CH}_{2}$ & 24.83 & $\mathrm{CH}_{2}$ & 26.96 & $\mathrm{CH}_{2}$ & 24.77 & $\mathrm{CH}_{2}$ \\
\hline 4 & 29.08 & $\mathrm{CH}_{2}$ & 29.04 & $\mathrm{CH}_{2}$ & 27.18 & $\mathrm{CH}_{2}$ & 29.12 & $\mathrm{CH}_{2}$ & 28.92 & $\mathrm{CH}_{2}$ \\
\hline 5 & 29.68 & $\mathrm{CH}_{2}$ & 29.28 & $\mathrm{CH}_{2}$ & 29.19 & $\mathrm{CH}_{2}$ & 31.39 & $\mathrm{CH}_{2}$ & 29.12 & $\mathrm{CH}_{2}$ \\
\hline 6 & 29.54 & $\mathrm{CH}_{2}$ & 29.11 & $\mathrm{CH}_{2}$ & 29.18 & $\mathrm{CH}_{2}$ & 29.51 & $\mathrm{CH}_{2}$ & 29.09 & $\mathrm{CH}_{2}$ \\
\hline 7 & 29.54 & $\mathrm{CH}_{2}$ & 29.18 & $\mathrm{CH}_{2}$ & 29.67 & $\mathrm{CH}_{2}$ & 29.64 & $\mathrm{CH}_{2}$ & 29.51 & $\mathrm{CH}_{2}$ \\
\hline 8 & 29.54 & $\mathrm{CH}_{2}$ & 31.52 & $\mathrm{CH}_{2}$ & 27.18 & $\mathrm{CH}_{2}$ & 29.51 & $\mathrm{CH}_{2}$ & 31.39 & $\mathrm{CH}_{2}$ \\
\hline 9 & 29.76 & $\mathrm{CH}_{2}$ & 130.12 & $\mathrm{CH}$ & 129.95 & $\mathrm{CH}$ & 29.40 & $\mathrm{CH}_{2}$ & 129.75 & $\mathrm{CH}$ \\
\hline 10 & 29.54 & $\mathrm{CH}_{2}$ & 129.63 & $\mathrm{CH}$ & 127.86 & $\mathrm{CH}$ & 28.40 & $\mathrm{CH}_{2}$ & 127.95 & $\mathrm{CH}$ \\
\hline 11 & 29.54 & $\mathrm{CH}_{2}$ & 31.93 & $\mathrm{CH}_{2}$ & 25.61 & $\mathrm{CH}_{2}$ & 129.73 & $\mathrm{CH}$ & 26.96 & $\mathrm{CH}_{2}$ \\
\hline 12 & 29.63 & $\mathrm{CH}_{2}$ & 29.71 & $\mathrm{CH}_{2}$ & 128.04 & $\mathrm{CH}$ & 129.81 & $\mathrm{CH}$ & 127.89 & $\mathrm{CH}$ \\
\hline 13 & 29.34 & $\mathrm{CH}_{2}$ & 29.38 & $\mathrm{CH}_{2}$ & 129.96 & $\mathrm{CH}$ & 28.90 & $\mathrm{CH}_{2}$ & 129.73 & $\mathrm{CH}$ \\
\hline 14 & 31.91 & $\mathrm{CH}_{2}$ & 29.67 & $\mathrm{CH}_{2}$ & 29.04 & $\mathrm{CH}_{2}$ & 29.09 & $\mathrm{CH}_{2}$ & 33.54 & $\mathrm{CH}_{2}$ \\
\hline 15 & 22.69 & $\mathrm{CH}_{2}$ & 29.54 & $\mathrm{CH}_{2}$ & 29.48 & $\mathrm{CH}_{2}$ & 29.51 & $\mathrm{CH}_{2}$ & 29.64 & $\mathrm{CH}_{2}$ \\
\hline 16 & 14.10 & $\mathrm{CH}_{3}$ & 31.93 & $\mathrm{CH}_{2}$ & 31.91 & $\mathrm{CH}_{2}$ & 33.75 & $\mathrm{CH}_{2}$ & 31.39 & $\mathrm{CH}_{2}$ \\
\hline 17 & - & - & 22.58 & $\mathrm{CH}_{2}$ & 22.58 & $\mathrm{CH}_{2}$ & 22.39 & $\mathrm{CH}_{2}$ & 22.50 & $\mathrm{CH}_{2}$ \\
\hline 18 & - & - & 14.10 & $\mathrm{CH}_{3}$ & 14.06 & $\mathrm{CH}_{3}$ & 13.55 & $\mathrm{CH}_{3}$ & 13.55 & $\mathrm{CH}_{3}$ \\
\hline $1^{\prime}$ & 173.18 & C & 172.75 & $\mathrm{C}$ & 172.75 & $\mathrm{C}$ & 172.41 & $\mathrm{C}$ & 172.41 & $\mathrm{C}$ \\
\hline $2^{\prime}$ & 68.86 & $\mathrm{CH}$ & 68.86 & $\mathrm{CH}$ & 68.86 & $\mathrm{CH}$ & 69.01 & $\mathrm{CH}$ & 69.01 & $\mathrm{CH}$ \\
\hline $3^{\prime}$ & 62.05 & $\mathrm{CH}_{2}$ & 62.05 & $\mathrm{CH}_{2}$ & 62.05 & $\mathrm{CH}_{2}$ & 61.87 & $\mathrm{CH}_{2}$ & 61.87 & $\mathrm{CH}_{2}$ \\
\hline
\end{tabular}

${ }^{\mathrm{a}} 1 \mathrm{D}$ APT, 2D HMBC, and ACD NMR program are used for interpretation. 
Table 6. ${ }^{1} \mathrm{H}$ NMR data of compounds $5 \mathbf{5 a}, \mathbf{5 b}$, and $\mathbf{5 c}$ (400 MHz, acetone- $\left.\mathrm{d}_{6}\right)$

\begin{tabular}{ll|ll|ll}
\hline \multicolumn{5}{c}{ Compounds $\left(\delta_{\mathrm{H}}: \mathrm{ppm}, J=\mathrm{Hz}\right)^{\mathrm{a}}$} \\
\hline & $\mathbf{5 a}(16: 0)$ & \multicolumn{2}{c}{$\mathbf{5 b}(18: 1, Z) \Delta^{9}(E)$} & \multicolumn{2}{c}{$\mathbf{5 c}(18: 0)$} \\
\hline $\mathrm{H}_{2}$ & $2.27, \mathrm{t}, J=7.4$ & $\mathrm{H}_{2}$ & $2.27, \mathrm{t}, J=7.4$ & $\mathrm{H}_{2}$ & $2.27, \mathrm{t}, J=7.4$ \\
$\mathrm{H}_{3}-\mathrm{H}_{15}$ & $1.20-1.60, \mathrm{~m}$ & $\mathrm{H}_{3}-\mathrm{H}_{7}$ & $1.20-1.60, \mathrm{~m}$ & $\mathrm{H}_{13^{\prime}}-\mathrm{H}_{17}$ & $1.20-1.60, \mathrm{~m}$ \\
$\mathrm{H}_{16}$ & $0.88, \mathrm{t}, J=7.2$ & $\mathrm{H}_{8}$ & $1.94-2.04, \mathrm{~m}$ & $\mathrm{H}_{18}$ & $0.88, \mathrm{t}, J=7.2$ \\
- & - & $\mathrm{H}_{9}-\mathrm{H}_{10}$ & $5.20-5.39, \mathrm{~m}$ & - & - \\
- & - & $\mathrm{H}_{11}$ & $1.94-2.04, \mathrm{~m}$ & - & - \\
- & - & $\mathrm{H}_{12^{-}-\mathrm{H}_{17}}$ & $1.20-1.60, \mathrm{~m}$ & - & - \\
- & - & $\mathrm{H}_{18}$ & $0.88, \mathrm{t}, J=7.2$ & - & - \\
$\mathrm{H}_{1^{\prime}}$ & $5.24-5.33, \mathrm{~m}$ & $\mathrm{H}_{1^{\prime}}$ & $5.24-5.33, \mathrm{~m}$ & $\mathrm{H}_{1^{\prime}}$ & $5.24-5.33, \mathrm{~m}$ \\
$\mathrm{H}_{2^{\prime}}$ & $1.60, \mathrm{~m}$ & $\mathrm{H}_{2}$ & $1.60, \mathrm{~m}$ & $\mathrm{H}_{2^{\prime}}$ & $1.60, \mathrm{~m}$ \\
$\mathrm{H}_{3^{\prime}}$ & $4.67-4.75, \mathrm{~m}$ & $\mathrm{H}_{3^{\prime}}$ & $4.67-4.75, \mathrm{~m}$ & $\mathrm{H}_{3^{\prime}}$ & $4.67-4.75, \mathrm{~m}$ \\
$\mathrm{H}_{4^{\prime}}$ & $1.40-1.60, \mathrm{~m}$ & $\mathrm{H}_{4^{\prime}}$ & $1.40-1.60, \mathrm{~m}$ & $\mathrm{H}_{4^{\prime}}$ & $1.40-1.60, \mathrm{~m}$ \\
$\mathrm{H}_{5^{\prime}}$ & $1.48, \mathrm{~m}$ & $\mathrm{H}_{5^{\prime}}$ & $1.48, \mathrm{~m}$ & $\mathrm{H}_{5^{\prime}}$ & $1.48, \mathrm{~m}$ \\
$\mathrm{H}_{6^{\prime}}$ & $1.50, \mathrm{~m}$ & $\mathrm{H}_{6^{\prime}}$ & $1.50, \mathrm{~m}$ & $\mathrm{H}_{6^{\prime}}$ & $1.50, \mathrm{~m}$ \\
$\mathrm{H}_{7^{\prime}}$ & $1.45-1.60, \mathrm{~m}$ & $\mathrm{H}_{7^{\prime}}$ & $1.45-1.60, \mathrm{~m}$ & $\mathrm{H}_{7^{\prime}}$ & $1.45-1.60, \mathrm{~m}$ \\
$\mathrm{H}_{8^{\prime}}$ & $1.45, \mathrm{~m}$ & $\mathrm{H}_{8^{\prime}}$ & $1.45, \mathrm{~m}$ & $\mathrm{H}_{8^{\prime}}$ & $1.45, \mathrm{~m}$ \\
$\mathrm{H}_{9^{\prime}}$ & $1.45-1.60, \mathrm{~m}$ & $\mathrm{H}_{9^{\prime}}$ & $1.45-1.60, \mathrm{~m}$ & $\mathrm{H}_{9^{\prime}}$ & $1.45-1.60, \mathrm{~m}$ \\
$\mathrm{H}_{10^{\prime}}$ & $1.60, \mathrm{~m}$ & $\mathrm{H}_{10^{\prime}}$ & $1.60, \mathrm{~m}$ & $\mathrm{H}_{10^{\prime}}$ & $1.60, \mathrm{~m}$ \\
$\mathrm{H}_{11^{\prime}}$ & $0.92, \mathrm{~d}, J=6.4$ & $\mathrm{H}_{11^{\prime}}$ & $0.92, \mathrm{~d}, J=6.4$ & $\mathrm{H}_{11^{\prime}}$ & $0.92, \mathrm{~d}, J=6.4$ \\
$\mathrm{H}_{12^{\prime}}$ & $0.92, \mathrm{~d}, J=6.4$ & $\mathrm{H}_{12^{\prime}}$ & $0.92, \mathrm{~d}, J=6.4$ & $\mathrm{H}_{12^{\prime}}$ & $0.92, \mathrm{~d}, J=6.4$ \\
$\mathrm{H}_{13^{\prime}}$ & $0.96, \mathrm{~d}, J=6.5$ & $\mathrm{H}_{13^{\prime}}$ & $0.96, \mathrm{~d}, J=6.5$ & $\mathrm{H}_{13^{\prime}}$ & $0.96, \mathrm{~d}, J=6.5$ \\
$\mathrm{H}_{14^{\prime}}$ & $0.86, \mathrm{~d}, J=6.6$ & $\mathrm{H}_{14^{\prime}}$ & $0.86, \mathrm{~d}, J=6.6$ & $\mathrm{H}_{14^{\prime}}$ & $0.86, \mathrm{~d}, J=6.6$ \\
$\mathrm{H}_{15^{\prime}}$ & $1.14, \mathrm{~d}, J=6.4$ & $\mathrm{H}_{15^{\prime}}$ & $1.14, \mathrm{~d}, J=6.4$ & $\mathrm{H}_{15^{\prime}}$ & $1.14, \mathrm{~d}, J=6.4$ \\
\hline
\end{tabular}

2D COSY, and ACD NMR program are used for interpretation

When we check the the MBC values, it was determined that $S$. aureus $30 \mu \mathrm{g} / \mathrm{mL}$, B. cereus 7.5 $\mu \mathrm{g} / \mathrm{mL}$, and M. smegmatis $0.46 \mu \mathrm{g} / \mathrm{mL}$ were found to have bactericide activity. Compounds $\mathbf{5 a - c}$ is highly effective at the probiotic bacteria group L. casei, low concentrations to other Gram-positive bacteria $(8.5-68.8 \mu \mathrm{g} / \mathrm{mL})$, and very low to the ARB positive bacteria group $M$. smegmatis has been determined that it has high efficacy in low concentrations $(<0.5 \mu \mathrm{g} / \mathrm{mL})$. Compounds $\mathbf{1}$ and $\mathbf{5 a - c}$ have a killing activity against $M$. smegmatis, suggesting their potential to become an antituberculosis agent. Compounds 1 and 5a-c generally have similar efficacy but are observed to be effective at lower concentrations against Gram-negative bacteria and yeast fungi. It is thought that isolates carry high efficacy at low concentrations and increase the probability of being a potential drug. It was determined that the compounds $\mathbf{4}$ and $\mathbf{2}$ were similar in terms of antimicrobial activity and were effective against all microorganisms (broad-spectrum) at low concentrations $(61.2-0.8 \mu \mathrm{g} / \mathrm{mL})$. When MBC values were examined, it was observed that they had microbicide (Bacteriocyte/fungicide) activity against all tested microorganisms (broad-spectrum) except forspore bacteria B. cereus and M. smegmatis. MIC value of compound 3 was found to have a $338.8 \mu \mathrm{g} / \mathrm{mL}$ against all tested microorganisms. The lack of antimicrobial activity of compound $\mathbf{3}$ showed that it could not be used as an antimicrobial agent due to the lack of MBC.

In the study, the antimicrobial activity of the endophyte Fusarium sp. obtained from the leaves of the honeysuckle plant was investigated and compared with $1 \%$ streptomycin sulfate [49]. It was mentioned that endophyte Fusarium solani isolated from Taxus baccata (Yew) has antimicrobial activity on many microorganisms [13]. In the literature, the raw extract of a Fusarium species isolated from the sea showed the antibacterial (Bacillus subtilis, Streptococcus mutans, Staphylococccus epidermidis, E. coli, P. aeroginosa, and K. pneumoniae) and antifungal (Candida rugosa, F. oxysporium, S. cerevisiae, Rhizopus oryzae and Aspergillus flavus) activities [11]. The best activity was observed against $E$. coli at a concentration of $200 \mu \mathrm{g} / \mathrm{mL}$. 
New metabolites produced by Fusarium oxysporum YP9B

Table 7. ${ }^{13} \mathrm{C}$ NMR data of compounds $5 \mathbf{a}, \mathbf{5 b}$, and $\mathbf{5 c}\left(100 \mathrm{MHz}\right.$, acetone- $\left.\mathrm{d}_{6}\right)$

\begin{tabular}{|c|c|c|c|c|c|c|}
\hline \multicolumn{7}{|c|}{ Compounds $\left(\delta_{\mathrm{C}}: \mathrm{ppm}\right)^{\mathrm{a}}$} \\
\hline No & \multicolumn{2}{|c|}{$5 a(16: 0)$} & \multicolumn{2}{|c|}{ 5b $(18: 1, Z) \Delta^{9}(E)$} & \multicolumn{2}{|c|}{ 5c $(18: 0)$} \\
\hline 1 & 174.18 & $\mathrm{C}$ & 168.94 & $\mathrm{C}$ & 170.19 & $\mathrm{C}$ \\
\hline 2 & 31.74 & $\mathrm{CH}_{2}$ & 31.74 & $\mathrm{CH}_{2}$ & 31.74 & $\mathrm{CH}_{2}$ \\
\hline 3 & 25.12 & $\mathrm{CH}_{2}$ & 26.95 & $\mathrm{CH}_{2}$ & 24.83 & $\mathrm{CH}_{2}$ \\
\hline 4 & 29.44 & $\mathrm{CH}_{2}$ & 29.03 & $\mathrm{CH}_{2}$ & 29.40 & $\mathrm{CH}_{2}$ \\
\hline 5 & 29.59 & $\mathrm{CH}_{2}$ & 29.24 & $\mathrm{CH}_{2}$ & 29.59 & $\mathrm{CH}_{2}$ \\
\hline 6 & 29.63 & $\mathrm{CH}_{2}$ & 29.12 & $\mathrm{CH}_{2}$ & 29.63 & $\mathrm{CH}_{2}$ \\
\hline 7 & 29.55 & $\mathrm{CH}_{2}$ & 29.03 & $\mathrm{CH}_{2}$ & 29.55 & $\mathrm{CH}_{2}$ \\
\hline 8 & 29.55 & $\mathrm{CH}_{2}$ & 31.74 & $\mathrm{CH}_{2}$ & 29.55 & $\mathrm{CH}_{2}$ \\
\hline 9 & 29.24 & $\mathrm{CH}_{2}$ & 129.70 & $\mathrm{CH}$ & 29.24 & $\mathrm{CH}_{2}$ \\
\hline 10 & 29.63 & $\mathrm{CH}_{2}$ & 127.92 & $\mathrm{CH}$ & 29.55 & $\mathrm{CH}_{2}$ \\
\hline 11 & 29.55 & $\mathrm{CH}_{2}$ & 31.74 & $\mathrm{CH}_{2}$ & 29.51 & $\mathrm{CH}_{2}$ \\
\hline 12 & 29.51 & $\mathrm{CH}_{2}$ & 29.60 & $\mathrm{CH}_{2}$ & 29.55 & $\mathrm{CH}_{2}$ \\
\hline 13 & 29.31 & $\mathrm{CH}_{2}$ & 29.26 & $\mathrm{CH}_{2}$ & 29.63 & $\mathrm{CH}_{2}$ \\
\hline 14 & 31.74 & $\mathrm{CH}_{2}$ & 29.63 & $\mathrm{CH}_{2}$ & 29.28 & $\mathrm{CH}_{2}$ \\
\hline 15 & 22.51 & $\mathrm{CH}_{2}$ & 29.40 & $\mathrm{CH}_{2}$ & 31.74 & $\mathrm{CH}_{2}$ \\
\hline 16 & 14.09 & $\mathrm{CH}_{3}$ & 31.98 & $\mathrm{CH}_{2}$ & 31.94 & $\mathrm{CH}_{2}$ \\
\hline 17 & - & - & 24.83 & $\mathrm{CH}_{2}$ & 22.51 & $\mathrm{CH}_{2}$ \\
\hline 18 & - & - & 13.63 & $\mathrm{CH}_{3}$ & 13.61 & $\mathrm{CH}_{3}$ \\
\hline $1^{\prime}$ & 74.65 & $\mathrm{CH}$ & 73.31 & $\mathrm{CH}$ & 73.31 & $\mathrm{CH}$ \\
\hline $2^{\prime}$ & 36.24 & $\mathrm{CH}$ & 36.24 & $\mathrm{CH}$ & 36.24 & $\mathrm{CH}$ \\
\hline $3^{\prime}$ & 62.17 & $\mathrm{CH}_{2}$ & 61.90 & $\mathrm{CH}_{2}$ & 62.03 & $\mathrm{CH}_{2}$ \\
\hline $4^{\prime}$ & 31.74 & $\mathrm{CH}_{2}$ & 31.74 & $\mathrm{CH}_{2}$ & 31.74 & $\mathrm{CH}_{2}$ \\
\hline $5^{\prime}$ & 29.63 & $\mathrm{CH}_{2}$ & 29.63 & $\mathrm{CH}_{2}$ & 29.63 & $\mathrm{CH}_{2}$ \\
\hline $6^{\prime}$ & 28.93 & $\mathrm{CH}$ & 28.93 & $\mathrm{CH}$ & 28.93 & $\mathrm{CH}$ \\
\hline $7^{\prime}$ & 31.74 & $\mathrm{CH}_{2}$ & 31.74 & $\mathrm{CH}_{2}$ & 31.74 & $\mathrm{CH}_{2}$ \\
\hline $8^{\prime}$ & 25.12 & $\mathrm{CH}_{2}$ & 25.12 & $\mathrm{CH}_{2}$ & 25.12 & $\mathrm{CH}_{2}$ \\
\hline $9^{\prime}$ & 36.24 & $\mathrm{CH}_{2}$ & 36.24 & $\mathrm{CH}_{2}$ & 36.24 & $\mathrm{CH}_{2}$ \\
\hline $10^{\prime}$ & 29.63 & $\mathrm{CH}$ & 29.63 & $\mathrm{CH}$ & 29.63 & $\mathrm{CH}$ \\
\hline $11^{\prime}$ & 19.61 & $\mathrm{CH}_{3}$ & 19.61 & $\mathrm{CH}_{3}$ & 19.61 & $\mathrm{CH}_{3}$ \\
\hline $12^{\prime}$ & 18.86 & $\mathrm{CH}_{3}$ & 18.86 & $\mathrm{CH}_{3}$ & 18.86 & $\mathrm{CH}_{3}$ \\
\hline $13^{\prime}$ & 17.94 & $\mathrm{CH}_{3}$ & 17.94 & $\mathrm{CH}_{3}$ & 17.94 & $\mathrm{CH}_{3}$ \\
\hline $14^{\prime}$ & 10.98 & $\mathrm{CH}_{3}$ & 10.98 & $\mathrm{CH}_{3}$ & 10.98 & $\mathrm{CH}_{3}$ \\
\hline $15^{\prime}$ & 17.98 & $\mathrm{CH}_{3}$ & 17.98 & $\mathrm{CH}_{3}$ & 17.98 & $\mathrm{CH}_{3}$ \\
\hline
\end{tabular}

${ }^{\mathrm{a}}$ 1D APT, 2D HMBC, HSQC, and ACD NMR program are used for interpretation.

Table 8a. Minimal Inhibition (MIC) concentration values of the isolated compounds 1-5 $(\mu \mathrm{g})$

\begin{tabular}{|c|c|c|c|c|c|c|c|c|c|c|c|c|c|c|}
\hline \multirow{3}{*}{ Comp. } & \multirow{3}{*}{$\begin{array}{l}\text { Stock } \\
\text { con. } \\
\mu \mathrm{g} / \mathrm{mL}\end{array}$} & \multicolumn{13}{|c|}{ Minimal Inhibition Concentration Values (MIC) } \\
\hline & & \multicolumn{4}{|c|}{ Gram (-) } & \multicolumn{5}{|c|}{ Gram (+) } & \multicolumn{4}{|c|}{ ARB+ Yeast Like Fungi } \\
\hline & & $E c$ & $Y p$ & $K p$ & $P a$ & $S a$ & $E f$ & $S m$ & $L c$ & $B c$ & $M s$ & $\mathrm{Ca}$ & $C t$ & $S c$ \\
\hline 1 & 4800 & 60 & 60 & 60 & 60 & 0.94 & 1.8 & 1.8 & 30 & 0.94 & 0.47 & 60 & 60 & 120 \\
\hline 2 & 2000 & 1.6 & 1.6 & 1.6 & 1.6 & 0.8 & 1.6 & 6.3 & 12.5 & 0.8 & 3.1 & 3.1 & 3.1 & 6.3 \\
\hline 3a-c & 27100 & 338.8 & 338.8 & 338.8 & 338.8 & 338.8 & 338.8 & 338.8 & 338.8 & 338.8 & 169.3 & 338.8 & 338.8 & 338.8 \\
\hline 4a-b & 9800 & 7.8 & 7.7 & 7.7 & 7.7 & 3.8 & 7.7 & 30.6 & 61.2 & 7.7 & 30.6 & 30.6 & 30.6 & 30.6 \\
\hline 5a-c & 22000 & 550 & 550 & 550 & 550 & 4.3 & 4.3 & 4.3 & 1100 & 2.1 & $<0.5$ & 1100 & 1100 & 1100 \\
\hline Cont. & - & - & - & - & - & - & - & - & - & - & - & - & - & - \\
\hline Amp. & & 10 & 18 & 18 & $>128$ & 10 & 35 & NT & NT & 15 & & & & \\
\hline Strep. & & & & & & & & & & & 4 & & & \\
\hline Fluc. & & & & & & & & & & & & $<8$ & $<8$ & $<8$ \\
\hline
\end{tabular}


Table 8b. Minimal Bactericidal (MBC) concentration values of the isolated compounds 1-5 $(\mu \mathrm{g})$ Stock Minimal Bactericidal Concentration Values (MBC)

\begin{tabular}{cllcccccccccccc} 
Comp. & con. & \multicolumn{4}{c}{ Gram (-) } & \multicolumn{4}{c}{ Gram (+) } & \multicolumn{5}{c}{ ARB+ Yeast Like Fungi } \\
\cline { 3 - 16 } & & $E c$ & $Y p$ & $K p$ & $P a$ & $S a$ & $E f$ & $S m$ & $L c$ & $B c$ & $M s$ & $C a$ & $C t$ & $S c$ \\
\hline $\mathbf{1}$ & 4800 & - & - & - & - & 30 & - & - & - & 7.5 & 0.5 & - & - & - \\
$\mathbf{2}$ & 2000 & 3.1 & 3.1 & 3.1 & 3.1 & 6.3 & 12.5 & 12.5 & 25 & - & - & 12.5 & 12.5 & 12.5 \\
$\mathbf{3 a - c}$ & 27100 & - & - & - & - & - & - & - & - & - & - & - & - & - \\
4a-b & 9800 & 15.3 & 61.3 & 15.3 & 15.3 & 30.6 & 61.3 & 61.3 & 245 & - & - & 61.3 & 61.3 & 61.3 \\
5a-c & 22000 & - & - & - & - & 68.8 & 34.3 & 8.5 & - & 17.1 & $<0.5$ & - & - & -
\end{tabular}

Control -

Ec.: Escherichia coli, Yp.: Yersinia pseudotuberculosis, Kp.: Klebsiella pneumonia, Pa.: Pseudomonas aeruginosa, Sa.: Staphylococcus aureus, Ef.: Enterococcus faecalis, Sm.: Streptococcus mutans, Lc.: Lactobacillus casei, Bc.: Bacillus cereus 702 Roma, and Ms.: Mycobacterium smegmatis, Ca.: Candida albicans, Ct.: Candida tropicalis, and Sc.: Saccharomyces cerevisiae. (-): Activity not observed; Amp.: Ampicillin, Strep.: Streptomycin, (-): Fluc.: Fluconazole.

\subsection{Determination of Cytotoxic, and Antiviral Activity}

The cytotoxic activities of an isolate obtained from $F$. oxysporum YP9B were tested against the VERO cell line, the kidney green monkey (Cercopithecus aetiops) epithelial cell, and the results are given in Table 9 and Figure 2.

Table 9. Cytotoxic effect on VERO cells for compounds 1-5 isolated from $F$. oxysporum YP9B

\begin{tabular}{ccccccccc}
\hline Comp. & Control & \multicolumn{7}{c}{ Fraction Concentration $(\boldsymbol{\mu M})$ and Live Cell Count } \\
\cline { 3 - 9 } & cell & $\mathbf{4 0}$ & $\mathbf{2 0}$ & $\mathbf{1 0}$ & $\mathbf{5}$ & $\mathbf{2 . 5}$ & $\mathbf{1 . 2 5}$ & $\mathbf{0 . 6 2 5}$ \\
\hline $\mathbf{1}$ & 100 & - & - & 6.758 & 31.594 & 58.720 & 85.677 & 145.925 \\
$\mathbf{2}$ & 100 & - & 9.931 & 8.209 & 44.789 & 65.974 & 72.471 & 87.215 \\
$\mathbf{3 a - c}$ & 100 & 6.488 & 7.368 & 53.510 & 91.782 & 93.783 & 98.913 & 109.516 \\
$\mathbf{4 a - b}$ & 100 & - & - & 7.157 & 59.346 & 95.046 & 97.089 & 97.974 \\
$\mathbf{5 a - c}$ & 100 & - & - & 7.021 & 6.7166 & 6.330 & 6.523 & 6.457 \\
\hline
\end{tabular}

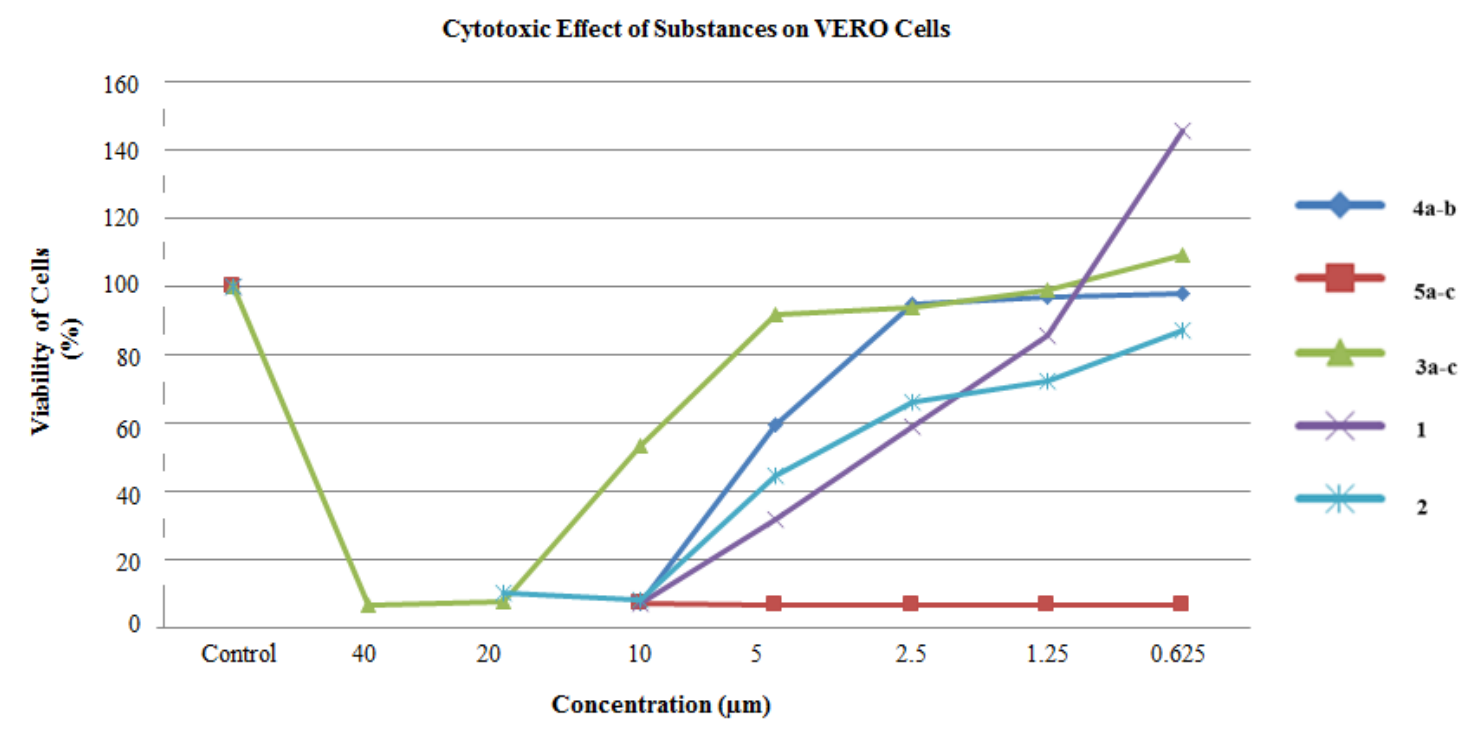

Figure 2. Cytotoxic effect of substances on VERO cells 
New metabolites produced by Fusarium oxysporum YP9B

Table 10. Antiviral effect of compounds 1-5 on HSV type-1

\begin{tabular}{cccccccccccc}
\hline \multirow{2}{*}{ Comp. } & \multirow{2}{*}{ Cell } & \multirow{2}{*}{ Virus } & \multirow{2}{*}{ Acyclovir } & \multicolumn{6}{c}{ Antiviral activity tests studied concentrations $(\boldsymbol{\mu M})$} \\
\cline { 6 - 13 } & & & 5 & 2.5 & 1.25 & 0.63 & 0.31 & 0.16 & 0.08 & 0.04 \\
\hline $\mathbf{1}$ & 100 & 33.70 & 92.83 & - & - & 38.46 & 39.90 & 48.88 & 44.24 & 42.30 & 42.74 \\
$\mathbf{2}$ & 100 & 33.70 & 92.83 & - & - & 37.36 & 43.46 & 37.11 & 36.56 & 35.52 & 23.69 \\
3a-c & 100 & 33.70 & 92.83 & 44.18 & 39.93 & 39.25 & 54.75 & 27.73 & 28.65 & - & - \\
4a-b & 100 & 33.70 & 92.83 & - & 50.11 & 54.96 & 39.68 & 40.03 & 30.77 & 45.92 & - \\
\hline
\end{tabular}

According to the results obtained, compounds 3a-c at five $\mu \mathrm{M}$ and below, compounds $\mathbf{4 a - b}$ at $2.5 \mu \mathrm{M}$ and below, and compounds 1 and 2 at $1.25 \mu \mathrm{M}$ and below were not showed cytotoxic to VERO cell lines. But, compound 5a-c had strong cytotoxic activity against the VERO cell line. Thus, antiviral activity tests for compounds 5a-c could not be performed. The antiviral activity of the isolated compounds 1-4 was determined using the HSV type-1 DNA virus that lysed VERO cells. The VERO cell line concentrations that remained alive compared to the virus control were higher than the virus control, as the antiviral activity value (Table $10, \mathbf{4 a - b} \leq 2.5 \mu \mathrm{M}$, and $\mathbf{1}$ and $\mathbf{2} \leq 1.25 \mu \mathrm{M}$ was effective in the antiviral activity that they could be used for antiviral activity tests. Thus two of the total five isolates showed antiviral activity. The result showed that compound $\mathbf{4 a - b}$ at $1.25 \mu \mathrm{M}$ and compound $\mathbf{1}$ at $0.312 \mu \mathrm{M}$ concentrations produced a partial increase in cell viability, and it was found to have antiviral activity for HSV Type-1.

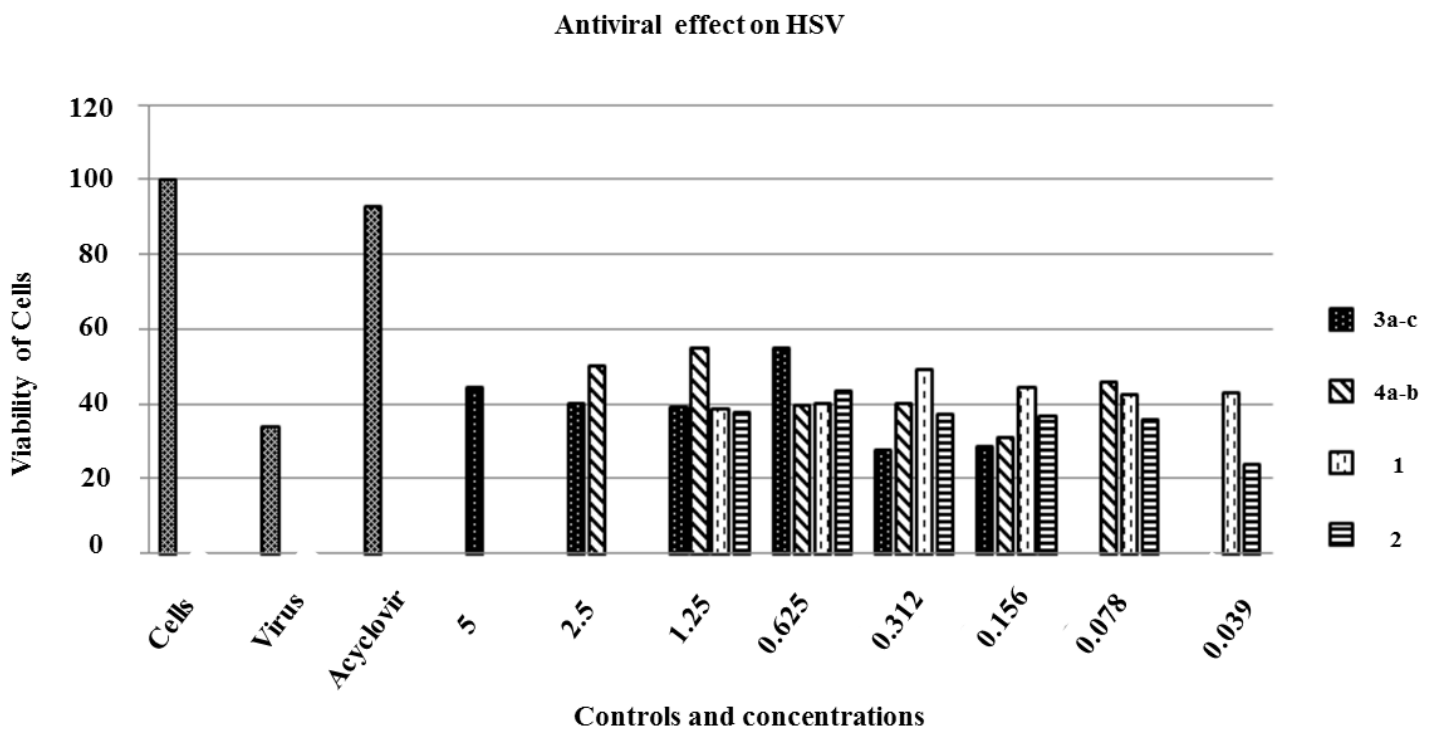

Figure 3. Antiviral activities of compounds 1-5 against HSV-1

The cytotoxic activities of the isolated compounds (1-5) were tested against the VERO cell line with the concentration of $5 \mu \mathrm{M}$ and below dilutions for compound $3,2.5 \mu \mathrm{M}$ and below dilutions for compound $4,1.25 \mu \mathrm{M}$ and below dilutions for compounds 1 and 2 (total six-fold dilution) was effective. Thus, they were used for antiviral activity tests. Compound $\mathbf{5}$ has a strong cytotoxic activity to VERO cells (Table 9, Figure 3). HSV type I, which is the DNA virus, was used for antiviral activity. HSV type-1 is a virus that lyses VERO cells. In determining antiviral activity, the number of cells that survive according to the VERO cell line's virus control and higher concentrations than the virus control is determined as the value of the antiviral activity. Accordingly, it was determined that compound 4 produced a partial increase in cell viability at concentrations of $1.25 \mu \mathrm{M}$, and compound $\mathbf{1}, 0.312 \mu \mathrm{M}$. 
The increase in cell viability of compound 3 at a concentration of $0.625 \mu \mathrm{M}$ was not considered significant since it could not be demonstrated in repeated experiments.

Cytotoxic activities of five isolate from $F$. oxysporum YP9B were tested using MCF-7, PC-3, and A549 cell lines. The compounds tested showed brow cytotoxic activity $\left(\mathrm{IC}_{50}\right)$ in the cell lines used (MCF-7, PC-3, and A549) (Table 11 and Figure 4).

Table 11. Cytotoxic activity values ( $\mathrm{IC}_{50}$ values) of the five isolate obtained from $F$. oxysporum YP9B on three cancer cell lines

\begin{tabular}{cccc}
\hline \multirow{2}{*}{ Comp. } & \multicolumn{3}{c}{ Cancer Cell Lines and $\mathbf{I C}_{\mathbf{5 0}}(\boldsymbol{\mu M}) \pm$ SD Values $(\boldsymbol{\mu M})$} \\
\cline { 2 - 4 } & $\mathbf{M C F}-\mathbf{7}$ & $\mathbf{P C}-\mathbf{3}$ & $\mathbf{A 5 4 9}$ \\
\hline $\mathbf{1}$ & $15.01 \pm 4.55$ & $19.13 \pm 0.68$ & $17.06 \pm 1.69$ \\
$\mathbf{2}$ & $790.33 \pm 32.56$ & $917.06 \pm 84.66$ & $905.35 \pm 82.24$ \\
$\mathbf{3 a - c}$ & $\mathrm{ni}$ & $\mathrm{ni}$ & $\mathrm{ni}$ \\
$\mathbf{4 a - b}$ & $\mathrm{ni}$ & $\mathrm{ni}$ & $\mathrm{ni}$ \\
$\mathbf{5 a - c}$ & $7.75 \pm 1.40$ & $17.75 \pm 0.65$ & $7.51 \pm 1.38$ \\
Doxorubicin & $0.053 \pm 0.004$ & $0.09 \pm 0.014$ & $17.75 \pm 5.61$ \\
\hline
\end{tabular}

ni: no inhibitions

Cytotoxic Activity on MCF-7, PC-3 and A549 Cell Lines

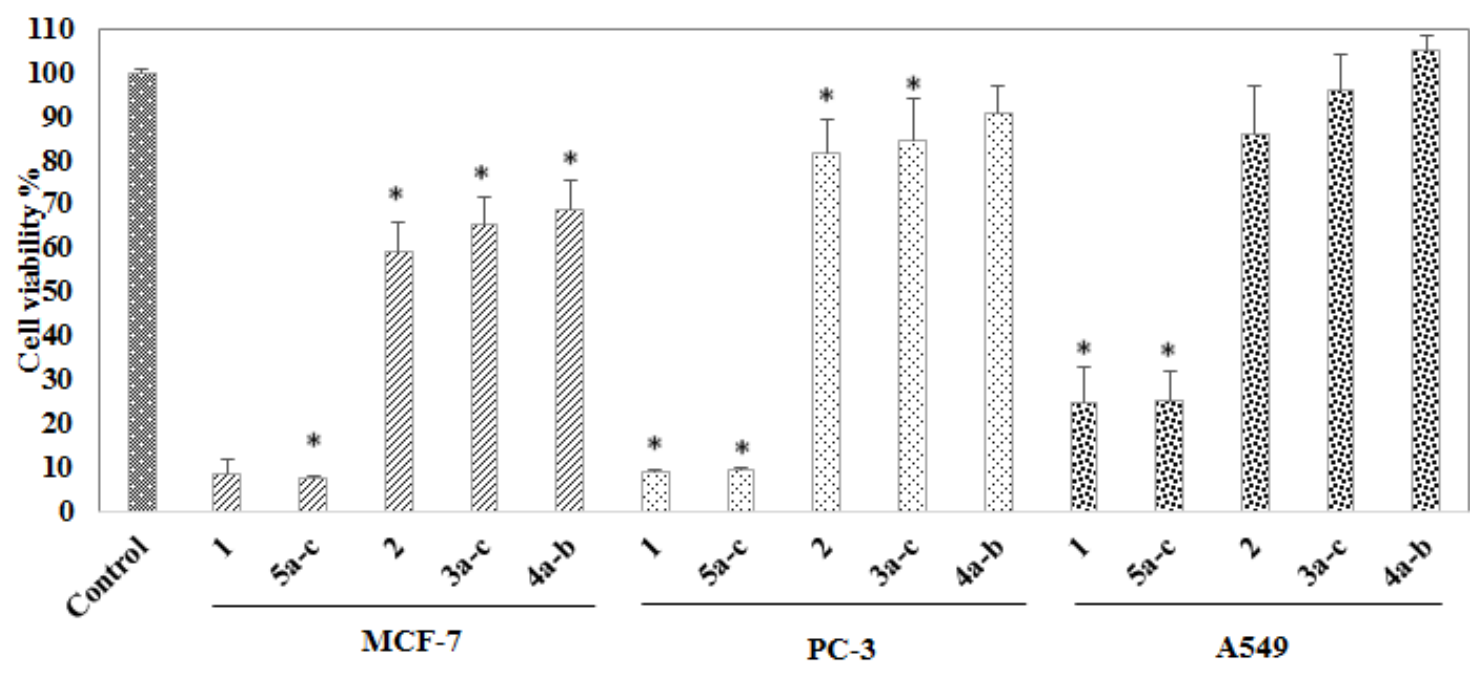

Figure 4. Cytotoxic activity of compounds at $100 \mu \mathrm{M}$ in MCF-7, PC-3, and A549 cell lines compared to the control group. The significance level of the differences between the groups and the control is defined by $* \mathrm{p}<0.05, * * \mathrm{p}<0.01$, and $* * * \mathrm{p}<0.0001$

As seen in Table 11, compounds 5a-c has shown the most potent anti-proliferative activity on all tested cell lines, notably. Comp compound $\mathbf{1}$ has exerted second potent activity on each cell line, while compounds 4a-b have the weakest inhibitory activity on MCF-7, PC-3, and A549 cell lines. Compared to other isolates, compounds 3a-c and $\mathbf{4 a - b}$ did not exert cytotoxicity on these cell lines. Furthermore, compounds 3a-c and 4a-b lead to an increase in tested cell lines' viability in a dosedependent manner $(2-1000 \mu \mathrm{M})$. Anti-cancer activity on MCF-7, PC-3, and A549 exposed to $100 \mu \mathrm{M}$ of test substances has been shown in Figure 4. Compounds 1, 5a-c to 2 were found to have anti-cancer activity against the breast cancer line MCF-7, the prostate cancer cell line PC-3, and the lung cancer cell line A549. Compounds 3a-c and 4a-b do not show cytotoxicity even at doses up to $1000 \mu \mathrm{M}$ against tested MCF-7, PC-3, and A549 cell lines. It has been determined that cell lines increase the 
viability in a dose-dependent manner. Therefore, these compounds (3a-c and 4a-b) did not show anticancer activities.

F. oxysporum is an important phytopathogenic fungus species that infects about 150 plant species and has a wide host range with biological activities [50-57]. Secondary metabolites obtained from Fusarium sp. have been found to vary from species to species [13-28], depending on the medium. Different secondary metabolites were isolated from the F. oxysporum strain in the literature, and their cytotoxic activities were tested against three cancer cell lines (PC-3, PANC-1, and A549) using the MTT method [40-41]. The efficacy of extracted Beauvericin to PC-3, PANC-1, and A549 cell lines was reported as $\mathrm{IC}_{50}: 49.5 \pm 3.8,47.2 \pm 2.9$, and $10.4 \pm 1.6 \mu \mathrm{M}$, respectively [5]. Beauvericin is also reported to have antibacterial activity against methicillin-resistant to $S$. aureus (MIC $=3.125 \mu \mathrm{g} / \mathrm{mL}$ ) and Bacillus subtilis (MIC $=3.125 \mu \mathrm{g} / \mathrm{mL}$ ) strains [5]. In another study, it was reported that ethyl acetate extract of $F$. oxysporum SS46 and $n$-hexane extracts of $F$. oxysporum SS50 isolates from solid rice medium was effective against HCT-8, MDA-MB435, and SF295 cancer cell lines in vitro [8]. In another study using the present technique, extracts from 14 different Fusarium species were fractionated by HPLC, aurofusarin, and bikaverin was observed as red pigments. They reported the antibacterial activity against Lactobacillus acidophilus at eight micrograms and against Bifidobacterium breve at 64 micrograms. However, there are no broad spectrums of antimicrobial, cytotoxicity, and antiviral activity for the secondary metabolites or strains producing metabolites related to the existing $F$. oxysporum strains [7]. The absolute configurations of the chiral carbon on the isolated compounds could not be established. Thus, no studies have been found in the literature regarding all these new compounds related to all of these works done in this manuscript.

\section{Acknowledgments}

This work was supported by TÜBİTAK (1002, 117S937), KTÜ, Faculty of Pharmacy, and RTE University, Department of Biology research facilities.

\section{Supporting Information}

Supporting information accompanies this paper on http://www.acgpubs.org/journal/recordsof-natural-products

\section{ORCID}

Gözde K1lıç: 0000-0003-1248-7912

Gonca Çelik: 0000-0002-4634-3354

Arif Bozdeveci: 0000-0002-0729-9143

İshak Erik: 0000-0002-9729-1013

Elif Öztürk: 0000-0002-0809-6145

Rengin Reis: 0000-0002-3484-2201

Hande Sipahi: 0000-0001-6482-3143

Merve Cora: 0000-0002-5956-9133

Şengül Alpay Karaoğlu: 0000-0003-1047-8350

Nurettin Yayl1: 0000-0003-4174-3014

\section{References}

[1] C. Placinta, J. D. Mello and A. Macdonald (1999). Fusarium mycotoxins: a review of global implications for animal health, welfare and productivity, Anim. Feed Sci. 80, 183-205.

[2] B. A. Summerell (2019). Resolving Fusarium: Current status of the genus, Annu. Rev. Phytopathol. 57, 323-339. 
[3] R. Dean, J. A. Van Kan, Z. A. Pretorius, K. E. Hammond-Kosack, A. D. Pietro, D. S. Jason, J. Rudd, M. Dickman, R. Kahmann, J. Ellis and G. D. Foster (2012). The top 10 fungal pathogens in molecular plant pathology, Mol. Plant. Pathol. 13, 414-430.

[4] W. Zhang and D. Zang (2013). Recent advances of secondary metabolites in genus Fusarium, Plant Physiol. 49, 201-216.

[5] Q. X. Wang, S. F. Li, F. Zhao, H. Q. Dai, C. S. Li, R. Ding, H. Gao, L. X. Zhang and H. Liu (2011). Chemical constituents from endophytic fungus Fusarium oxysporum, Fitoterapia. 82, 777-781.

[6] J. Breinhold, S. Ludvigsen, B. R. Rassing, C. N. Rosendahl, S. E. Nielsen and C. E. Olsen (1997). Oxysporidinone: a novel, antifungal N-methyl-4-hydroxy-2-pyridone from Fusarium oxysporum, J. Nat. Prod. 60, 33-35.

[7] T. E. Sondergaard, M. Fredborg, A. M. O. Christensen, S. K. Damsgaard, N. F. Kramer, H. Giese and J. L. Sørensen (2016). Fast screening of antibacterial compounds from Fusaria, Toxins, 8, 1-9.

[8] A. M. Nascimento, R. Conti, I. C. C. Turatti, B. C. Cavalcanti, L. V. Costa-Lotufo, C. Pessoa, M. O. Moraes, V. Manfrim, J. S. Toledo, A. K. Cruz and M.T. Pupo (2012). Bioactive extracts and chemical constituents of two endophytic strains of Fusarium oxysporum, Rev. Bras. Farmacog. 22, 1276-1281.

[9] C. E. P. Sanhueza and M. C. Degrossi (2004). Moniliformin, A Fusarium mycotoxin, Rev. Mex. Micol. 19, 102-112.

[10] A. Waskiewicz, P. Golinski, Z. Karolewski, L. Irzykowska, J. Bocianowski, M. Kostecki and Z. Weber (2010). Formation of fumonisins and other secondary metabolites by Fusarium oxysporum and $F$. proliferatum: a comparative study, Food Addit. Contam. 27, 608-615.

[11] J. Swathi, K. M. Sowjanya, K. Narendr and A. K. Satya (2013). Bioactivity assay of an 1solated marine Fusarium sp, J. BioSci. Biotechnol. 5, 179-186.

[12] H. Zhang, C. Ruan, X. Bai, M. Zhang, S. Zhu and Y. Jiang (2016). Isolation and identification of the antimicrobial agent beauvericin from the endophytic Fusarium oxysporum 5-19 with NMR and ESIMS/MS, Biomed. Res. Int. Article ID 1084670, 4.

[13] K. Tayung, B. P. Barik, D. K. Jha and D. C. Deka (2011). Identification and characterization of antimicrobial metabolite from an endophytic fungus, Fusarium solani isolated from bark of Himalayan yew, Mycosphere. 2, 203-213.

[14] A. Kour, A. S. Shawl, S. Rehman, P. Sultan, P. H. Qazi, P. Suden, R. K. Khajuria and V. Verma (2008). Isolation and identification of an endophytic strain of Fusarium oxysporum producing podophyllotoxin from Juniperus recurva, World J. Microbiol. Biotechnol. 24, 1115-1121.

[15] X. L. Liu, K. H. Huang, J. Z. Zhou, L. Meng, Y. Wang and L. X. Zhang (2012). Identification and antibacterial characteristics of an endophytic fungus Fusarium oxysporum from Lilium lancifolium, Lett. Appl. Microbiol. 55, 399-406.

[16] S. Shweta, S. Zuehlke, B. T. Ramesha, V. Priti, P. Mohana Kumar, G. Ravikanth, M. Spiteller, R. Vasudeva and R. U. Shaanker (2010). Endophytic fungal strains of Fusarium solani, from Apodytes dimidiata E. Mey. Ex Arn (Icacinaceae) produce camptothecin, 10-hydroxycamptothecin and 9methoxycamptothecin, Phytochemistry. 71, 117-122.

[17] T. Bartók, Á. Szécsi, A. Szekeres, Á. Mesterházy and M. Bartók (2006). Detection of new fumonisin mycotoxins and fumonisin-like compounds by reversed-phase high-performance liquid chromatography/electrospray ionization ion trap mass spectrometry, Rapid Commun. Mass Spectrom. 20, 2447-2462.

[18] T. Bartók, L. Tölgyesi, A. Szekeres, M. Varga, R. Bartha, Á. Szécsi and Á. Mesterházy (2010). Detection and characterization of twenty-eight isomers of fumonisin B1 (FB1) mycotoxin in a solid rice culture infected with Fusarium verticillioides by reversed-phase high-performance liquid chromatography/electrospray ionization time-of-flight and ion trap mass spectrometry, Rapid Commun. Mass Spectrom. 24, 35-42.

[19] V. Nenkep, K. Yun, D. Zhang, H. D. Choi, J. S. Kang and B. W. Son (2010). Induced production of bromomethylchlamydosporols A and B from the marine-derived fungus Fusarium tricinctum, J. Nat. Prod. 73, 2061-2063.

[20] J. Chełkowski, K. Gromadzka, Ł. Stępień, L. Lenc, M. Kostecki and F. Berthiller (2012). Fusarium species, zearalenone and deoxynivalenol content in preharvest scabby wheat heads from Poland, World Mycotoxin J. 5, 133-141.

[21] A. Logrieco, A. Moretti, A. Ritieni, M. F. Caiaffa and L. Macchia (2002). Advances in microbial toxin research and its biotechnological exploitation, Beauvericin: Chemistry, biology and significance, Kluwer Academic, New York, pp 23-30. 
New metabolites produced by Fusarium oxysporum YP9B

[22] H. F. Mohamed (2012). Molecular analysis and anti-cancer properties of two identified isolates, Fusarium solani and Emericella nidulans isolated from Wady El-Natron soil in Egypt against Caco-2 (ATCC) cell line, Asian Pac. J. Trop. Biomed. 2, 863-869.

[23] S. V. Von Bargen, O. Martinez, I. Schadock, A. M. Eisold, M. Gossmann and C. Büttner (2009). Genetic variability of phytopathogenic Fusarium proliferatum associated with crown rot in Asparagus officinalis, J. Phytopathol.157, 446-456.

[24] Ł. Stępień, K. Gromadzka and J. Chełkowski (2012). Polymorphism of mycotoxin biosynthetic genes among Fusarium equiseti isolates from Italy and Poland, J. Appl. Genet. 53, 227-236.

[25] L. Gao, J. Huang and J. Li (2007). Fermentation conditions of Sinopodophyllum hexandrum endophytic fungus on production of podophyllotoxin, Food and Fermentation Industries. 33, 28-32.

[26] S. Kusari, S. Zühlke and M. Spiteller (2009). An endophytic fungus from Camptotheca acuminata that produces camptothecin and analogues, J. Nat. Prod. 72, 2-7

[27] R. F. Vesonder and C.W. Hesseltine (1981). Metabolites of Fusarium.In Fusarium: Diseases, Biology and Taxonomy, ed: P.E. Nelson, T.A. Toussoun, R. J. Cook, Penn. State Univ. Press, Park/London, pp. 350-364.

[28] Y. Kasap (2018). Isolation of Trichoderma spp. strains with Fusarium from tomato seedlings and soil in Rize and determination of biocontrol activities, Master Thesis, Institute of Science. Recep Tayyip Erdoğan University, Rize, Turkiye.

[29] G. Veyisoğlu (2019). Diagnosis, characterization, and antimicrobial properties of Fusarium oxysporum YP9B, Master Thesis, Institute of Science. Recep Tayyip Erdoğan University, Rize, Turkiye.

[30] G.S. D. Rani and M. K. Naik (2008). Cultural, morphological and pathogenic diversity among Fusarium isolates causing wilt of Chilli, J. Soil Biol. Ecol. 28, 82-100.

[31] M. Tamura, A. Uyama and N. Mochizuki (2011). Development of a multi-mycotoxin analysis in beerbased drinks by a modified QuEChERS method and ultra-high-performance liquid chromatography coupled with tandem mass spectrometry, Anal. Sci. 27, 629-635.

[32] Y. Taghdi, R. Hermosa, S. Domínguez, M. B. Rubio, H. Essalmani, C. Nicolás and E. Monte (2015). Effectiveness of composts and Trichoderma strains for control of Fusarium wilt of tomato, Phytopathol. Mediterr. 54, 232-240.

[33] N. Isik S. Aslan Erdem and M. Kartal (2019). Investigation the fatty acid profile of commercial black cumin seed oils and seed oil capsules: Application to real samples, J. Chem. Metrol. 13, 53-60.

[34] N. T. Chung, L. T. Huong and I. A. Ogunwande (2020). Antimicrobial, larvicidal activities and composition of the leaf Essential oil of Magnolia coco (Lour.) DC, Rec. Nat. Prod. 14, 372-377.

[35] G. Renda, Y. Kalaycı, B. Korkmaz, Ş. Alpay Karaoglu and N. Yaylı (2017). Chemical Composition and antimicrobial activity of the essential oils of five Scrophularia L. species from Turkey, Rec. Nat. Prod. 11, 521-531.

[36] S. Fandaklı, N. Yaylı, N. Kahriman, E. Uzunalioğlu, N. Ulaş Çolak, S. Yıldırım and A. Yaşar (2019). The chemical composition of volatile oil and biological activity of Rhododendron caucasicum, Rec. Nat. Prod. 13, 316-323.

[37] N. Kahriman, B. Yaylı, Ş. Alpay Karaoğlu and N.Yaylı (2012). Chemical constituents and antimicrobial activity of the essential oil from Vicia dadianorum extracted by hydro and microwave distillations, Rec. Nat. Prod. 6, 49-56.

[38] I. Wiegand, K. Hilpert and R. E. W. Hancock (2008). Agar and broth dilution methods to determine the minimal inhibitory concentration (MIC) of antimicrobial substances, Nat. Protoc. 3, 163-175.

[39] G. L. Woods, B. A. Brown-Elliott, E. P. Desmond, G. S. Hall, L. Heifets, G. E Pfyffer, J. C. Ridderhof, R.J. Wallace, N. C. Warren and F. Witebsky (2003). Susceptibility testing of Mycobacteria nocardiae, and other aerobic actinomycetes; Approved Standard, NCCLS document, M24-A. 23, 1-61.

[40] Kilic, G. Topcu, A. C. Goren, Z. Aydogmus, A. Karagoz, Y. K. Yildiz and I. Aslan (2020). Ent-kaurene diterpenoids from Sideritis lycia with antiviral and cytotoxic activities, Rec. Nat. Prod. 14, 256-268.

[41] H. Şenol, K. Çokuludağ, A. Sena Aktaş, S. Atasoy, A. Dağ and G. Topçu (2020). Synthesis of new fatty acid derivatives of oleanane and ursane triterpenoids and investigation of their in vitro cytotoxic effects on 3 T3 fibroblast and PC3 prostate cancer cell lines, Org. Commun. 13, 114-126.

[42] W. Tsutomu, I. Mie, M. Toshio and U. Akira (1990). Sesquiterpene glycosides from Ixeris debilis and Ixeris repens, Phytochemistry. 29, 3217-3224.

[43] P. Muller, N. P. Buu-Hoi and R. Rips (1959). $\beta$-Cyanoethylation of phenoxazine and 7H-benzo[c] phenothiazine, Eur. J. Org. Chem. 24, 1699-1701.

[44] G. Frangatos, G. Kohan and F. L. Chubb (1960). The synthesis of 10-substituted phenoxazines, Can. J. Chem. 38, 1021-1025. 
[45] M. R. Stillings, S. Freeman, P. L. Myers, M. J. Readhead, A. P Welbourn, M. J. Rance and D. C. Atkinson (1985). Substituted 5H-dibenz[b,g]-1,4-oxazocines and related amino acids with anti-inflammatory activity, J. Med. Chem. 28, 225-233.

[46] P. Mestichelli, M. J. Scott, W. R. J. D Galloway, J. Selwyn, J. S. Parker and D. R. Spring (2013). Concise copper-catalyzed synthesis of tricyclic biaryl ether-linked aza-heterocyclic ring systems, Org. Lett. 15, 5448-5451.

[47] Y. Yang, I. Chung, S. Kim and A. Ahmad (2014). New sesterterpene diolyl butanoate from rice straw of Oryza sativa, Asian J. Chem. 26, 7792-7794,

[48] G. Tel-Cayan, A. Muhammad, M. E. Duru, M. Ozturk, A. Adhikari and A. Turkoglu (2016). A new fatty acid ester from an edible mushroom Rhizopogon luteolus, Nat. Prod. Res. 30, 2258-2264.

[49] H. Zhang, X. Sun and C. Xu (2016). Antimicrobial activity of endophytic funngus Fusarium sp., Arch. Biol. Sci. 68, 25-30.

[50] L. Xu, L. Zhou, J. Zhao, W. Jiang and L. Xu (2008). Recent studies on the antimicrobial compounds produced by plant endophytic fungi, Nat. Prod. Res. Develop. 20, 731-740.

[51] S. F. Musavi and R. M. Balakrishnan (2014). A Study on the antimicrobial potentials of an endophytic fungus Fusarium oxysporum NFX 06, J. Med. Biol. Eng. 3, 162-166.

[52] V. Manon Mani, P. Shanmuga, M. S. Dhaylini and K. Preethi (2015). Antioxidant and antimicrobial evaluation of bioactive pigment from Fusarium sp. isolated from stressed environment, Int. Curr. Microbiol. App. Sci. 4, 1147-1158.

[53] R. G. Shu, F. W. Wang, Y. M. Yang, Y. X. Liu and R. X. Tan (2004). Antibacterial and xanthine oxidase inhibitory cerebrosides from Fusarium sp. IFB-121, an endophytic fungus in Quercus variabilis, Lipids. 39, 667-73.

[54] P. B. Ratnaweera, E. D. Silva, D. E. Williams and R. J. Andersen (2015). Antimicrobial activities of endophytic fungi obtained from the arid zone invasive plant Opuntia dillenii and the isolation of equisetin, from endophytic Fusarium sp., BMC Complement Altern. Med. 15, 1-7.

[55] M. V. Tejesvi, D. R. Segura, K. M. Schnorr, D. Sandvang, S. Mattila, P. B. Olsen, S. Neve, T. Kruse, H. H. Kristensen and A. M. Pirttilä (2013). An antimicrobial peptide from endophytic Fusarium tricinctum of Rhododendron tomentosum Harmaja, Fungal Divers. 60, 153.

[56] R. R. Hateet, T. M. Muhsin and K. J. Humadi (2014). Antibacterial activities secondary metabolites from endophytic fungus Fusarium solani, J. Basra. Res. 40, 94-101.

[57] J. Zhan, A. M. Burns, M. X. Liu, S. H. Faeth and A. A. Gunatilaka (2007). Search for cell motility and angiogenesis inhibitors with potential anti-cancer activity: beauvericin and other constituents of two endophytic strains of Fusarium oxysporum, J. Nat. Prod. 70, 227-232.

$$
\begin{aligned}
& \text { A C G } \\
& \text { publications } \\
& \text { (C) } 2021 \text { ACG Publications }
\end{aligned}
$$

